\title{
Robust Linear Regression: Optimal Rates in Polynomial Time
}

\author{
Ainesh Bakshi \\ abakshi@cs.cmu.edu \\ Carnegie Mellon University \\ Pittsburgh, USA
}

\author{
Adarsh Prasad \\ adarshp@andrew.cmu.edu \\ Carnegie Mellon University \\ Pittsburgh, USA
}

\begin{abstract}
We obtain robust and computationally efficient estimators for learning several linear models that achieve statistically optimal convergence rate under minimal distributional assumptions. Concretely, we assume our data is drawn from a $k$-hypercontractive distribution and an $\epsilon$-fraction is adversarially corrupted. We then describe an estimator that converges to the optimal least-squares minimizer for the true distribution at a rate proportional to $\epsilon^{2-2 / k}$, when the noise is independent of the covariates. We note that no such estimator was known prior to our work, even with access to unbounded computation. The rate we achieve is information-theoretically optimal and thus we resolve the main open question in Klivans, Kothari and Meka [COLT'18].

Our key insight is to identify an analytic condition that serves as a polynomial relaxation of independence of random variables. In particular, we show that when the moments of the noise and covariates are negatively-correlated, we obtain the same rate as independent noise. Further, when the condition is not satisfied, we obtain a rate proportional to $\epsilon^{2-4 / k}$, and again match the informationtheoretic lower bound. Our central technical contribution is to algorithmically exploit independence of random variables in the "sum-of-squares" framework by formulating it as the aforementioned polynomial inequality.
\end{abstract}

\section{CCS CONCEPTS}

- Theory of computation $\rightarrow$ Semidefinite programming; Convex optimization; $\bullet$ Mathematics of computing $\rightarrow$ Multivariate statistics.

\section{KEYWORDS}

robust linear regression, heavy-tailed distributions, sum-of-squares

\section{ACM Reference Format:}

Ainesh Bakshi and Adarsh Prasad. 2021. Robust Linear Regression: Optimal Rates in Polynomial Time. In Proceedings of the 53rd Annual ACM SIGACT Symposium on Theory of Computing (STOC '21), fune 21-25, 2021, Virtual, Italy. ACM, New York, NY, USA, 14 pages. https://doi.org/10.1145/3406325. 3451001

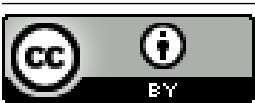

This work is licensed under a Creative Commons Attribution International 4.0 License. STOC '21, fune 21-25, 2021, Virtual, Italy

(C) 2021 Copyright held by the owner/author(s).

ACM ISBN 978-1-4503-8053-9/21/06.

https://doi.org/10.1145/3406325.3451001

\section{INTRODUCTION}

While classical statistical theory has focused on designing statistical estimators assuming access to i.i.d. samples from a nice distribution, estimation in the presence of adversarial outliers has been a challenging problem since it was formalized by Huber [28]. A long and influential line of work in high-dimensional robust statistics has since focused on studying the trade-off between sample complexity, accuracy and more recently, computational complexity for basic tasks such as estimating mean, covariance [8-10, 15$17,35,37,54]$, moment tensors of distributions [35] and regression $[14,20,31,33,43,46]$.

Regression continues to be extensively studied under various models, including realizable regression (no noise), true linear models (independent noise), asymmetric noise, agnostic regression and generalized linear models (see [57] and references therein). In each model, a variety of distributional assumptions are considered over the covariates and the noise. As a consequence, there exist innumerable estimators for regression achieving various trade-offs between sample complexity, running time and rate of convergence. The presence of adversarial outliers adds yet another dimension to design and compare estimators.

Seminal works on robust regression focused on designing nonconvex loss functions, including M-estimators [29], Theil-Sen estimators [51, 56], R-estimators [30], Least-Median-Squares [49] and S-estimators [48]. These estimators have desirable statistical properties under disparate assumptions, yet remain computationally intractable in high dimensions. Further, recent works show that it is information-theoretically impossible to design robust estimators for linear regression without distributional assumptions [33].

An influential recent line of work showed that when the data is drawn from the well studied and highly general class of $h y$ percontractive distributions (see Definition 1.3), there exist robust and computationally efficient estimators for regression [22, 33, 43]. Several families of natural distributions fall into this category, including Gaussians, strongly log-concave distributions and product distributions on the hypercube. However, both estimators converge to the the true hyperplane (in $\ell_{2}$-norm) at a sub-optimal rate, as a function of the fraction of corrupted points.

Given the vast literature on ad-hoc and often incomparable estimators for high-dimensional robust regression, the central question we address in this work is as follows:

\section{Does there exist a unified approach to design robust and computationally efficient estimators achieving optimal rates for all linear regression models under mild distributional assumptions?}

We address the aforementioned question by introducing a framework to design robust estimators for linear regression when the input is drawn from a hypercontractive distribution. Our estimators 
converge to the true hyperplanes at the information-theoretically optimal rate (as a function of the fraction of corrupted data) under various well-studied noise models, including independent and agnostic noise. Further, we show that our estimators can be computed in polynomial time using the sum-of-squares convex hierarchy.

We note that, despite decades of progress, prior to our work, estimators achieving optimal convergence rate in terms of the fraction of corrupted points were not known, even with independent noise and access to unbounded computation.

\subsection{Our Results}

We begin by formalizing the regression model we work with. In classical regression, we assume $\mathcal{D}$ is a distribution over $\mathbb{R}^{d} \times \mathbb{R}$ and for a vector $\Theta \in \mathbb{R}^{d}$, the least-squares loss is given by $\operatorname{err}_{\mathcal{D}}(\Theta)=$ $\mathbb{E}_{x, y \sim \mathcal{D}}\left[\left(y-x^{\top} \Theta\right)^{2}\right]$. The goal is to learn $\Theta^{*}=\arg \min _{\Theta} \operatorname{err}_{\mathcal{D}}(\Theta)$. We assume sample access to $\mathcal{D}$, and given $n$ i.i.d. samples, we want to obtain a vector $\Theta$ that approximately achieves optimal error, $\operatorname{err}_{\mathcal{D}}\left(\Theta^{*}\right)$.

In contrast to the classical setting, we work in the strong contamination model. Here, an adversary has access to the input samples and is allowed to corrupt an $\epsilon$-fraction arbitrarily. Note, the adversary has access to unbounded computation and has knowledge of the estimators we design. We note that this is the most stringent corrupt model and captures Huber contamination, additive corruption, label noise, agnostic learning etc (see [19]). Formally,

Model 1.1 (Robust Regression Model). Let $\mathcal{D}$ be a distribution over $\mathbb{R}^{d} \times R$ such that the marginal distribution over $\mathbb{R}^{d}$ is centered and has covariance $\Sigma^{*}$ and let $\Theta^{*}=\arg \min _{\Theta} \mathbb{E}_{x, y \sim \mathcal{D}}\left[(y-\langle\Theta, x\rangle)^{2}\right]$ be the optimal hyperplane for $\mathcal{D}$. Let $\left\{\left(x_{1}^{*}, y_{1}^{*}\right),\left(x_{2}^{*}, y_{2}^{*}\right), \ldots\left(x_{n}^{*}, y_{n}^{*}\right)\right\}$ be $n$ i.i.d. random variables drawn from $\mathcal{D}$. Given $\epsilon>0$, the robust regression model $\mathcal{R}_{\mathcal{D}}\left(\epsilon, \Sigma^{*}, \Theta^{*}\right)$ outputs a set of n samples $\left\{\left(x_{i}, y_{i}\right)\right\}_{i \in[n]}$ such that for at least $(1-\epsilon) n$ points $x_{i}=x_{i}^{*}$ and $y_{i}=y_{i}^{*}$. The remaining $\epsilon n$ points are arbitrary, and potentially adversarial w.r.t. the input and estimator.

A natural starting point is to assume that the marginal distribution over the covariates (the $x$ 's above) is heavy-tailed and has bounded, finite covariance. However, we show that there is no robust estimator in this setting, even when the linear model has no noise and the uncorrupted points lie on a line.

Theorem 1.2 (Bounded Covariance does not suffice, TheOREM 6.3 INFORMAL). For all $\epsilon>0$, there exist two distributions $\mathcal{D}_{1}, \mathcal{D}_{2}$ over $\mathbb{R}^{d} \times \mathbb{R}$ such that $d_{T V}\left(\mathcal{D}_{1}, \mathcal{D}_{2}\right) \leqslant \epsilon$ and the marginal distribution over the covariates has bounded covariance, denoted by $\Sigma^{2}=\Theta(1)$, yet $\left\|\Sigma^{1 / 2}\left(\Theta_{1}-\Theta_{2}\right)\right\|_{2}=\Omega(1)$, where $\Theta_{1}$ and $\Theta_{2}$ are the optimal hyperplanes for $\mathcal{D}_{1}$ and $\mathcal{D}_{2}$.

The aforementioned result precludes any statistical estimator that converges to the true hyperplane as the fraction of corrupted points tends to 0 . Therefore, we strengthen the distributional assumption consider hypercontractive distributions instead.

Definition $1.3((C, k)$-Hypercontractivity). A distribution $\mathcal{D}$ over $\mathbb{R}^{d}$ is $(C, k)$-hypercontractive for an even integer $k \geqslant 4$, if for all

$$
\begin{aligned}
& r \in[k / 2] \text {, for all } v \in \mathbb{R}^{d}, \\
& \qquad \underset{x \sim \mathcal{D}}{\mathbb{E}}\left[\langle v, x-\mathbb{E}[x]\rangle^{2 r}\right] \leqslant \underset{x \sim \mathcal{D}}{\mathbb{E}}\left[C\langle v, x-\mathbb{E}[x]\rangle^{2}\right]^{r}
\end{aligned}
$$

Remark 1.4. Hypercontractivity captures a broad class of distributions, including Gaussian distributions, uniform distributions over the hypercube and sphere, affine transformations of isotropic distributions satisfying Poincare inequalities [34] and strongly logconcave distributions. Further, hypercontractivity is preserved under natural closure properties like affine transformations, products and weighted mixtures [36]. Further, efficiently computable estimators appearing in this work require certifiable-hypercontractivity (Definition 3.5), a strengthening that continues to capture aforementioned distribution classes.

In this work we focus on the rate of convergence of our estimators to the true hyperplane, $\Theta^{*}$, as a function of the fraction of corrupted points, denoted by $\epsilon$. We measure convergence in both parameter distance ( $\ell_{2}$-distance between the hyperplanes) and least-squares error on the true distribution $\left(\operatorname{err}_{\mathcal{D}}\right)$.

We introduce a simple analytic condition on the relationship between the noise (marginal distribution over $y-x^{\top} \Theta^{*}$ ) and covariates (marginal distribution over $x$ ) that can be considered as a proxy for independence of $y-x^{\top} \Theta^{*}$ and $x$ :

Definition 1.5 (Negatively Correlated Moments). Given a distribution $\mathcal{D}$ over $\mathbb{R}^{d} \times \mathbb{R}$, such that the marginal distribution on $\mathbb{R}^{d}$ is $\left(c_{k}, k\right)$-hypercontractive, the corresponding regression instance has negatively correlated moments if for all $r \leqslant k$, and for all $v$,

$$
\begin{aligned}
& \underset{x, y \sim \mathcal{D}}{\mathbb{E}}\left[\langle v, x\rangle^{r}\left(y-x^{\top} \Theta^{*}\right)^{r}\right] \\
& \leqslant O(1) \underset{x \sim \mathcal{D}}{\mathbb{E}}\left[\langle v, x\rangle^{r}\right] \underset{x, y \sim \mathcal{D}}{\mathbb{E}}\left[\left(y-x^{\top} \Theta^{*}\right)^{r}\right] .
\end{aligned}
$$

Informally, the negatively correlated moments condition can be viewed as a polynomial relaxation of independence of random variables. Note, it is easy to see that when the noise is independent of the covariates, the above definition is satisfied.

Remark 1.6. We show that when this condition is satisfied by the true distribution, $\mathcal{D}$, we obtain rates that match the information theoretically optimal rate in a true linear model, where the noise (marginal distribution over $y-x^{\top} \Theta^{*}$ ) is independent of the covariates (marginal distribution over $x$ ). Further, when this condition is not satisfied, we show that there exist distributions for which obtaining rates matching the true linear model is impossible.

When the distribution over the input is hypercontractive and has negatively correlated moments, we obtain an estimator achieving rate proportional to $\epsilon^{1-1 / k}$ for parameter recovery. Further, our estimator can be computed efficiently. Thus, our main algorithmic result is as follows:

Theorem 1.7 (Robust Regresssion with Negatively CorreLATEd Noise, Theorem 5.1 Informal). Given $\epsilon>0, k \geqslant 4$, and $n \geqslant(d \log (d))^{O(k)}$ samples from $\mathcal{R}_{\mathcal{D}}\left(\epsilon, \Sigma^{*}, \Theta^{*}\right)$, such that $\mathcal{D}$ is $(c, k)$-certifiably hypercontractive and has negatively correlated moments, there exists an algorithm that runs in $n^{O(k)}$ time and outputs an estimator $\tilde{\Theta}$ such that with high probability,

$$
\left\|\left(\Sigma^{*}\right)^{1 / 2}\left(\Theta^{*}-\tilde{\Theta}\right)\right\|_{2} \leqslant O\left(\epsilon^{1-1 / k}\right)\left(\operatorname{err}_{\mathcal{D}}\left(\Theta^{*}\right)^{1 / 2}\right)
$$


and,

$$
\operatorname{err}_{\mathcal{D}}(\tilde{\Theta}) \leqslant\left(1+O\left(\epsilon^{2-2 / k}\right)\right) \operatorname{err}_{\mathcal{D}}\left(\Theta^{*}\right)
$$

Remark 1.8. We note that prior work does not draw a distinction between the independent and dependent noise models. In comparison (see Table 1), Klivans, Kothari and Meka [33] obtained a sub-optimal least-squares error scales proportional to $\epsilon^{1-2 / k}$. For the special case of $k=4$, Prasad et. al. [43] obtain least squares error proportional to $O\left(\epsilon \kappa^{2}(\Sigma)\right)$, where $\kappa$ is the condition number. In very recent independent work Zhu, Jiao and Steinhardt [59] obtained a sub-optimal least-squares error scales proportional to $\epsilon^{2-4 / k}$.

Further, we show that the rate we obtained in Theorem 1.7 is information-theoretically optimal, even when the noise and covariates are independent:

Theorem 1.9 (Lower Bound For Independent Noise, TheoREM 6.1 INFORMAL ). For any $\epsilon>0$, there exist two distributions $\mathcal{D}_{1}, \mathcal{D}_{2}$ over $\mathbb{R}^{2} \times \mathbb{R}$ such that the marginal distribution over $\mathbb{R}^{2}$ has covariance $\Sigma$ and is $(c, k)$-hypercontractive for both distributions, and yet $\left\|\Sigma^{1 / 2}\left(\Theta_{1}-\Theta_{2}\right)\right\|_{2}=\Omega\left(\epsilon^{1-1 / k} \sigma\right)$, where $\Theta_{1}, \Theta_{2}$ are the optimal hyperplanes for $\mathcal{D}_{1}$ and $\mathcal{D}_{2}, \sigma=\max \left(\operatorname{err}_{\mathcal{D}_{1}}\left(\Theta_{1}\right)\right.$, err $\left.\mathcal{D}_{2}\left(\Theta_{2}\right)\right)$ and the noise is uniform over $[-\sigma, \sigma]$. Further, $\left|\operatorname{err}_{\mathcal{D}_{1}}\left(\Theta_{2}\right)-\operatorname{err}_{\mathcal{D}_{1}}\left(\Theta_{1}\right)\right|$ $=\Omega\left(\epsilon^{2-2 / k} \sigma^{2}\right)$.

Next, we consider the setting where the noise is allowed to arbitrary, and need not have negatively correlated moments with the covariates. A simple modification to our algorithm and analysis yields an efficient estimator that obtains rate proportional to $\epsilon^{1-2 / k}$ for parameter recovery.

Corollary 1.10 (Robust Regresssion with Dependent Noise, Corollary 4.2 Informal). Given $\epsilon>0, k \geqslant 4$ and $n \geqslant d O(k)$ samples from $\mathcal{R}_{\mathcal{D}}\left(\epsilon, \Sigma^{*}, \Theta^{*}\right)$, such that $\mathcal{D}$ is $(c, k)$-certifiably hypercontractive, there exists an algorithm that runs in $n^{O(k)}$ time and outputs an estimator $\tilde{\Theta}$ such that with probability 9/10,

$$
\left\|\left(\Sigma^{*}\right)^{1 / 2}\left(\Theta^{*}-\tilde{\Theta}\right)\right\|_{2} \leqslant O\left(\epsilon^{1-2 / k}\right)\left(\operatorname{err}_{\mathcal{D}}\left(\Theta^{*}\right)^{1 / 2}\right)
$$

and,

$$
\operatorname{err}_{\mathcal{D}}(\tilde{\Theta}) \leqslant\left(1+O\left(\epsilon^{2-4 / k}\right)\right) \operatorname{err}_{\mathcal{D}}\left(\Theta^{*}\right)
$$

Further, we show that the dependence on $\epsilon$ is again informationtheoretically optimal:

Theorem 1.11 (Lower Bound for Dependent Noise, TheoREM 6.2 INFORMAL). For any $\epsilon>0$, there exist two distributions $\mathcal{D}_{1}, \mathcal{D}_{2}$ over $\mathbb{R}^{2} \times \mathbb{R}$ such that the marginal distribution over $\mathbb{R}^{2}$ has covariance $\Sigma$ and is $(c, k)$-hypercontractive for both distributions, and yet $\left\|\Sigma^{1 / 2}\left(\Theta_{1}-\Theta_{2}\right)\right\|_{2}=\Omega\left(\epsilon^{1-2 / k} \sigma\right)$, where $\Theta_{1}, \Theta_{2}$ be the optimal hyperplanes for $\mathcal{D}_{1}$ and $\mathcal{D}_{2}$, and $\sigma=\max \left(\operatorname{err}_{\mathcal{D}_{1}}\left(\Theta_{1}\right)\right.$, err $\left.\mathcal{D}_{2}\left(\Theta_{2}\right)\right)$. Further, $\left|\operatorname{err}_{\mathcal{D}_{1}}\left(\Theta_{2}\right)-\operatorname{err}_{\mathcal{D}_{1}}\left(\Theta_{1}\right)\right|=\Omega\left(\epsilon^{2-4 / k} \sigma^{2}\right)$.

Applications for Gaussian Covariates. The special case where the marginal distribution over $x$ is Gaussian has received considerable interest recently $[18,21]$. We note that our estimators extend to the setting of Gaussian covariates, since the uniform distribution
Table 1: Comparison of convergence rate (for least-squares error) achieved by various computationally efficient estimators for Robust Regression, when the underlying distribution is $\left(c_{k}, k\right)$-hypercontractive.

\begin{tabular}{|l|c|c|}
\hline Estimator & $\begin{array}{l}\text { Independent } \\
\text { Noise }\end{array}$ & $\begin{array}{l}\text { Arbitrary } \\
\text { Noise }\end{array}$ \\
\hline $\begin{array}{l}\text { Prasad et. al. [43], } \\
\text { Diakonikolas et. al. [18] }\end{array}$ & $\begin{array}{l}\epsilon \kappa^{2} \\
\text { (only } k=4)\end{array}$ & $\begin{array}{l}\epsilon \kappa^{2} \\
\text { (only } k=4)\end{array}$ \\
\hline $\begin{array}{l}\text { Klivans, Kothari and Meka } \\
\text { [33] }\end{array}$ & $\epsilon^{1-2 / k}$ & $\epsilon^{1-2 / k}$ \\
\hline $\begin{array}{l}\text { Zhu, Jiao and Steinhardt } \\
{[59]}\end{array}$ & $\epsilon^{2-4 / k}$ & $\epsilon^{2-4 / k}$ \\
\hline $\begin{array}{l}\text { Our Work } \\
\text { Thm 1.7, Cor } 1.10\end{array}$ & $\epsilon^{2-2 / k}$ & $\epsilon^{2-4 / k}$ \\
\hline $\begin{array}{l}\text { Lower Bounds } \\
\text { Thm 1.9, Thm 1.11 }\end{array}$ & $\epsilon^{2-2 / k}$ & $\epsilon^{2-4 / k}$ \\
\hline
\end{tabular}

over samples from $\mathcal{N}(0, \Sigma)$ are $(O(k), O(k))$-certifiably hypercontractive for all $k$ (see Section 5 in Kothari and Steurer [36]). As a consequence, instantiating Corollary 1.10 with $k=\log (1 / \epsilon)$ yields the following:

Corollary 1.12 (Robust Regression with Gaussian CovariATES). Given $\epsilon>0$ and $n \geqslant(d \log (d))^{O(\log (1 / \epsilon))}$ samples from $\mathcal{R}_{\mathcal{N}}\left(\epsilon, \Sigma^{*}, \Theta^{*}\right)$, such that the marginal distribution over the $x$ 's is $\mathcal{N}\left(0, \Sigma^{*}\right)$, there exists an algorithm that runs in $n^{O(\log (1 / \epsilon)}$ time and outputs an estimator $\tilde{\Theta}$ such that with high probability,

$$
\left\|\left(\Sigma^{*}\right)^{1 / 2}\left(\Theta^{*}-\tilde{\Theta}\right)\right\|_{2} \leqslant O(\epsilon \log (1 / \epsilon))\left(\operatorname{err}_{\mathcal{N}}\left(\Theta^{*}\right)\right)^{1 / 2}
$$

and,

$$
\operatorname{err}_{\mathcal{N}}(\tilde{\Theta}) \leqslant\left(1+O\left((\epsilon \log (1 / \epsilon))^{2}\right)\right) \operatorname{err}_{\mathcal{N}}\left(\Theta^{*}\right)
$$

We note that our estimators obtain the rate matching recent work for Gaussians, albeit in quasi-polynomial time. In comparison, Diakonikolas, Kong and Stewart [21] obtain the same rate in polynomial time, when the noise is independent of the covariates. We note that obtaining the optimal rate for Gaussian covariates (shaving the additional $\log (1 / \epsilon)$ factor) remains an outstanding open question.

\subsection{Related Work}

Robust Statistics. Computationally efficient estimators for robust statistics in high dimension have been extensively studied, following the initial work on robust mean estimation [15,37]. We focus on literature regarding robust regression and sum-of-squares. We refer the reader to recent surveys and theses for an extensive discussion of the literature on robust statistics [19, 39, 45, 53].

Robust Regression. Computationally efficient estimators for robust linear regression were proposed by [14, 22, 33, 43]. While [43] and [14] obtained estimators for the more general case of distributions with bounded 4th moment. However, their estimators suffer an error of $O\left(\operatorname{err}_{D}\left(\Theta^{*}\right) \epsilon \kappa^{2}(\Sigma)\right)$, where $\kappa(\Sigma)$ is the condition number of the covariance matrix of $X$. Hence, these estimators don't obtain the optimal dependence on $\epsilon$ in the negatively correlated noise setting, and also suffer an additional condition number 
dependence in the dependent noise setting. [22] obtained improved bounds under the restrictive assumption that $X$ is distributed according to a Gaussian. [33] obtained polynomial-time estimators for distributions with certifiably bounded distributions, however, their estimators obtain a sub-optimal error of $O\left(\operatorname{err}_{D}\left(\Theta^{*}\right) \epsilon^{1-2 / k}\right)$. In very recent and independent work, Zhu, Jiao and Steinhardt [59] obtained polynomial time estimators for the dependent noise setting, but their estimators are sub-optimal for the negatively correlated setting.

There has been significant work in more restrictive noise models as well. For instance, a series of works $[5,6,55]$ consider a noise model where the adversary is only allowed to corrupt the labels and obtain consistent estimators in this regime (error goes to zero with more samples). In comparison, our estimators do not obtain For a comprehensive overview we refer the reader to references in the aforementioned papers.

Sum-of-Squares Algorithms. In recent years, there has been a significant progress in applying the Sum-of-Squares framework to design efficient algorithms for several fundamental computational problems. Starting with the work of Barak, Kelner and Steurer [4], sum-of-squares algorithms have the best known running time for dictionary learning and tensor decomposition [27, 40, 50], optimizing random tensors over the sphere [7] and refuting CSPs below the spectral threshold [44].

In the context of high-dimensional estimation, sum-of-squares algorithms achieved state-of-the-art performance for robust moment estimation [36], robust regression[33], robustly learning mixtures of spherical [26, 34] and arbitrary Gaussians [2, 13], heavy-tailed estimation $[11,25]$ and list-decodable variants of these problems $[1,12,31,46,47]$.

Concurrent Work. We note that a statistical estimator achieving rate proportional to $\epsilon^{1-1 / k}$ can be obtained from combining ideas in [58] and [59 $]^{1}$. However, this approach remains computationally intractable. Finally, Cherapanamjeri et al. [11] consider the special case of $k=4$ and obtain nearly linear sample complexity and running time. However, their running time and rate incurs a condition number dependence. Further, their rate scales proportional to $\epsilon^{1 / 2}$, even when the noise is indpendent of the covariates (as opposed to $\left.\epsilon^{3 / 4}\right)$.

We emphasize that the bottleneck in all prior and concurrent work remains algorithmically exploiting the independence of the noise and covariates, which we achieve via the negatively correlated moments condition (Definition 1.5).

\section{TECHNICAL OVERVIEW}

In this section, we provide an overview of our approach, the new algorithmic ideas we introduce and the corresponding technical challenges. At a high level, we build on several recent works that study Sum-of-Squares relaxations for solving algorithmic problems arising in robust statistics Following the proofs-to-algorithms paradigm arising from the aforementioned works, we show that given two distributions over regression instances that are close in total variation distance (definition 3.1), any hyperplane minimizing

${ }^{1}$ We thank Banghua Zhu, Jiantao Jiao, and Jacob Steinhardt for communicating their observation to us. the least-squares loss on one distribution must be close (in $\ell_{2}$ distance) to any other hyperplane minimizing the loss on the second distribution.

This information-theoretic statement immediately yields a robust estimator achieving optimal rate, albeit given access to unbounded computation. To see this, let $\mathcal{D}_{1}$ be the uniform distribution over $n$ i.i.d samples from the true distribution, and $\mathcal{D}_{2}$ be the uniform distribution over $n$ corrupted samples from $\mathcal{R}_{\mathcal{D}}\left(\epsilon, \Sigma^{*}, \Theta^{*}\right)$, denoted by $\mathcal{D}_{2}$. It is easy to check that the total variation distance between $\mathcal{D}_{1}$ and $\mathcal{D}_{2}$ is at most $\epsilon$. Therefore, the two hyperplanes must be close in $\ell_{2}$ norm. In order to make this strategy algorithmic, we show that we can distilled a set of polynomial constraints from the information theoretic proof and can efficiently optimize over them using the Sum-of-Squares framework. We note that we crucially require several new constraints, including the gradient condition and the negatively-correlated moments condition (see Section 2.1), which did not appear in any prior works. We describe each step in more detail subsequently.

\subsection{Total Variation Closeness Implies Hyperplane Closeness}

Consider two distributions $\mathcal{D}_{1}$ and $\mathcal{D}_{2}$ over $\mathbb{R}^{d} \times \mathbb{R}$ such that the total variation distance between $\mathcal{D}_{1}$ and $\mathcal{D}_{2}$ is $\epsilon$ and the marginals for both distributions over $\mathbb{R}^{d}$ are $\left(c_{k}, k\right)$-hypercontractive and have covariance $\Sigma$. Ignoring computational and sample complexity concerns, we can obtain the optimal hyperplanes corresponding to each distribution. Note, these hyperplanes need not be unique and are simply charecterized as minimzers of the least-squares loss : for $i \in\{1,2\}$,

$$
\Theta_{i}=\arg \min _{\Theta} \underset{x, y \sim \mathcal{D}_{i}}{\mathbb{E}}\left[\left(y-x^{\top} \Theta\right)^{2}\right]
$$

Our central contribution is to obtain an information theoretic proof that the optimal hyperplanes are indeed close in scaled $\ell_{2}$ norm, i.e.

$$
\begin{aligned}
\left\|\Sigma^{1 / 2}\left(\Theta_{1}-\Theta_{2}\right)\right\|_{2} \leqslant & O\left(\epsilon^{1-1 / k}\right)\left(\underset{x, y \sim \mathcal{D}_{1}}{\mathbb{E}}\left[\left(y-x^{\top} \Theta_{1}\right)^{2}\right]^{1 / 2}\right. \\
& \left.+\underset{x, y \sim \mathcal{D}_{2}}{\mathbb{E}}\left[\left(y-x^{\top} \Theta_{2}\right)^{2}\right]^{1 / 2}\right)
\end{aligned}
$$

We refer the reader to Theorem 4.1 for a precise statement. Further, we show that the $\epsilon^{1-1 / k}$ dependence can be achieved even when the noise is not completely independent of the covariates but satisfies an analytic condition which we refer to as negatively correlated moments (see Definition 1.5). We provide an outline of the proof as it illustrates the techniques we introduced in this work.

Coupling and Decoupling. We begin by considering a maximal coupling, $\mathcal{G}$, between distributions $\mathcal{D}_{1}$ and $\mathcal{D}_{2}$ such that they disagree on at most an $\epsilon$-measure support ( $\epsilon$-fraction of the points for a discrete distribution). Let $(x, y) \sim \mathcal{D}_{1}$ and $\left(x^{\prime}, y^{\prime}\right) \sim \mathcal{D}_{2}$. Then, observe for any vector $v$,

$$
\begin{aligned}
\left\langle v, \Sigma\left(\Theta_{1}-\Theta_{2}\right)\right\rangle & =\left\langle v, \underset{\mathcal{G}}{\mathbb{E}}\left[x x^{\top}\right]\left(\Theta_{1}-\Theta_{2}\right)\right\rangle \\
& =\underset{\mathscr{G}}{\mathbb{E}}\left[\left\langle v, x\left(x^{\top} \Theta_{1}-y\right)\right\rangle\right]+\underset{\mathcal{G}}{\mathbb{E}}\left[\left\langle v, x\left(y-x^{\top} \Theta_{2}\right)\right\rangle\right]
\end{aligned}
$$


While the first term in Equation (2) depends completely on $\mathcal{D}_{1}$, the second term requires using the properties of the maximal coupling. Since $1=1_{(x, y)=\left(x^{\prime}, y^{\prime}\right)}+1_{(x, y) \neq\left(x^{\prime}, y^{\prime}\right)}$, we can rewrite the second term in Equation (2) as follows:

$$
\begin{aligned}
\underset{\mathcal{G}}{\mathbb{E}}\left[\left\langle v, x\left(y-x^{\top} \Theta_{2}\right)\right\rangle\right]=\underset{\mathcal{G}}{\mathbb{E}}\left[\left\langle v, x^{\prime}\left(y^{\prime}-\left(x^{\prime}\right)^{\top} \Theta_{2}\right)\right\rangle 1_{\left.(x, y)=\left(x^{\prime}, y^{\prime}\right)\right]}\right. \\
+\underset{\mathcal{G}}{\mathbb{E}}\left[\left\langle v, x\left(y-x^{\top} \Theta_{2}\right)\right\rangle 1_{\left.(x, y) \neq\left(x^{\prime}, y^{\prime}\right)\right]}\right.
\end{aligned}
$$

With a bit of effort, we can combine Equations (2) and (3), and upper bound them as follows (see Theorem 4.1 for details):

$$
\begin{aligned}
& \left\langle v, \Sigma\left(\Theta_{1}-\Theta_{2}\right)\right\rangle \leqslant \\
& O(1)(\underbrace{\underset{\mathcal{G}}{\mathbb{E}}\left[\left\langle v, x\left(x^{\top} \Theta_{1}-y\right)\right\rangle\right]}_{(\mathrm{i})}+\underbrace{\underset{\mathcal{G}}{\mathbb{E}}\left[\left\langle v, x^{\prime}\left(\left(x^{\prime}\right)^{\top} \Theta_{2}-y^{\prime}\right)\right\rangle\right]}_{(\mathrm{ii})} \\
& +\underset{\mathcal{G}}{\mathbb{E}}\left[\left\langle v, x\left(y-x^{\top} \Theta_{1}\right)\right\rangle 1_{\left.(x, y) \neq\left(x^{\prime}, y^{\prime}\right)\right]}\right. \\
& +\underset{\mathcal{G}}{\mathbb{E}}\left[\left\langle v, x^{\prime}\left(y^{\prime}-\left(x^{\prime}\right)^{\top} \Theta_{2}\right)\right\rangle 1_{\left.(x, y) \neq\left(x^{\prime}, y^{\prime}\right)\right]}\right)
\end{aligned}
$$

Observe, since we have a maximal coupling, the last two terms appearing in Equation (4) are non-zero only on an $\epsilon$-measure support. To bound them, we decouple the indicator using Hölder's inequality,

$$
\begin{aligned}
& \underset{\mathcal{G}}{\mathbb{E}}\left[\left\langle v, x\left(y-x^{\top} \Theta_{1}\right)\right\rangle 1_{(x, y) \neq\left(x^{\prime}, y^{\prime}\right)}\right] \\
& \leqslant \mathbb{E}\left[1_{(x, y) \neq\left(x^{\prime}, y^{\prime}\right)}\right]^{\frac{k-1}{k}} \mathbb{E}\left[\langle v, x\rangle^{k}\left(y-x^{\top} \Theta_{1}\right)^{k}\right]^{\frac{1}{k}} \\
& \leqslant \epsilon^{1-1 / k} \cdot \underbrace{\mathbb{E}\left[\langle v, x\rangle^{k}\left(y-x^{\top} \Theta_{1}\right)^{k}\right]^{\frac{1}{k}}}
\end{aligned}
$$

where we used the maximality of the coupling $\mathcal{G}$ to bound $\mathbb{E}[1\{(x, y)$ $\left.\left.\neq\left(x^{\prime}, y^{\prime}\right)\right\}\right] \leqslant \epsilon$. The above analysis can be repeated verbatim for the second term in (4) as well. Going forward, we focus on bounding terms (i), (ii) and (iii).

Gradient Conditions. To bound terms (i) and (ii) in Equation (4), we crucially rely on gradient information provided by the leastsquares objective. Concretely, a key observation in our informationtheoretic proof is that the candidate hyperplanes are locally optimal: given least-squares loss, for $i \in\{1,2\}$ for all vectors $v$,

$$
\left\langle\nabla \underset{x, y \sim \mathcal{D}_{i}}{\mathbb{E}}\left[\left(y-x^{\top} \Theta_{i}\right)^{2}\right], v\right\rangle=\underset{x, y \sim \mathcal{D}_{i}}{\mathbb{E}}\left[\left\langle v, x x^{\top} \Theta_{i}-x y\right\rangle\right]=0
$$

where $\Theta_{1}$ and $\Theta_{2}$ are the optimal hyperplanes for $\mathcal{D}_{1}$ and $\mathcal{D}_{2}$ respectively. Therefore, both (i) and (ii) are identically 0 . It remains to bound (iii).

Independence and Negatively Correlated Moments. We observe that term (iii) can be interpreted as the $k$-th order correlation between the distribution of the covariates projected along $v$ and the distribution of the noise in the linear model. Here, we observe that if the linear model satisfies the negatively correlated moments condition (Definition 1.5), we can decouple the expectation and bound each term independently:

$$
\mathbb{E}\left[\langle v, x\rangle^{k}\left(y-x^{\top} \Theta_{1}\right)^{k}\right]^{1 / k} \leqslant \mathbb{E}\left[\langle v, x\rangle^{k}\right]^{1 / k} \mathbb{E}\left[\left(y-x^{\top} \Theta_{1}\right)^{k}\right]^{1 / k}
$$

Observe, when the underlying linear model has independent noise, Equation (6) follows for any $k$. We thus crucially exploit the structure of the noise and require a considerably weaker notion than independence. Further, if the negatively correlated moments property is not satisfied, we can use Cauchy-Schwarz to decouple the expectation in Equation (6) and incur a $\epsilon^{1-2 / k}$ dependence (see Corollary 4.2). Conceptually, we emphasize that the negatively correlated moments condition may be of independent interest to design estimators that exploit independence in various statistics problems.

Hypercontractivity. To bound the RHS in Equation (6), we use our central distributional assumption of hypercontractive $k$-th moments (Definition 1.3) of the covariates :

$$
\mathbb{E}\left[\langle v, x\rangle^{k}\right]^{1 / k} \leqslant \sqrt{c_{k}} \mathbb{E}\left[\langle v, x\rangle^{2}\right]^{1 / 2}=\sqrt{c_{k}}\langle v, \Sigma v\rangle^{1 / 2}
$$

We can bound the noise similarly, by assuming that the noise is hypercontractive and this considerably simplifies our statements. However, hypercontractivity of the noise is not a necessary assumption and prior work indeed incurs a term proportional to the $k$-th moment of the noise. Assuming boundedness of the regression vectors, Klivans, Kothari and Meka [33] obtained a uniform upper bound on $k$-th moment of the noise by truncating large samples. We note that the same holds for our estimators and we refer the reader to Section 5.2.3 in their paper. Finally, substituting $v=\Theta_{1}-\Theta_{2}$ and rearranging, completes the information-theoretic proof.

We note that our approach already differs from prior work $[33,43,58]$ and to our knowledge, we obtain the first information theoretic proof that being $\epsilon$-close in TV distance implies that the optimal hyperplanes are $O\left(\epsilon^{1-1 / k}\right)$ close in $\ell_{2}$ distance. Next, we use this proof as motivation to construct a robust estimator. We do this by explicitly enforcing the gradient condition, negatively correlated moments, and hypercontractivity as constraints in the description of the robust estimator. In contrast, prior work only uses hypercontractivity or the gradient information in the analysis of their robust estimators. We describe these details below.

\subsection{Proofs to Inefficient Algorithms: Distilling Constraints}

Given an $\epsilon$-corrupted sample generated by Model 1.1, a natural approach to design a robust (albeit inefficient) estimator is to search over all subsets of size $(1-\epsilon) n$, minimize the least squares objective on each one and output the hyperplane that achieves the minimal least-squares objective. Klivans, Kothari and Meka [33] implicitly analyze this estimator and show that it obtains rate $\epsilon^{1 / 2-1 / k}$, which is sub-optimal. Instead, we search over all subsets of size $(1-\epsilon) n$ and in addition to minimizing the least-squares objective, we check if the uniform distribution over the samples is hypercontractive, the corresponding hyperplane satisfies the gradient condition and that the distribution over the covariates and noise satisfies the 
negatively correlated moments condition. Then, picking the hyperplane that achieves minimal least-squares cost and satisfies the aforementioned constraints obtains the optimal rate.

Since we work in the strong contamination model, where the adversary is allowed to both add and delete samples, there may not be any i.i.d. subset of $(1-\epsilon) n$ samples and thus enforcing constraints directly on the samples does not suffice. Instead, we create variables $2 n$ variables denoted by $\left\{\left(x_{1}^{\prime}, y_{1}^{\prime}\right), \ldots\left(x_{n}^{\prime}, y_{n}^{\prime}\right)\right\}$ that serve as a proxy for the uncorrupted samples. We can now enforce constraints on the variables with impunity since a there exists a feasible assignment, namely the uncorrupted samples. With the goal of obtaining an efficiently computable estimator, we restrict to enforcing only polynomial constraints, instead of arbitrary ones.

Intersection Constraints. The discrete analogue of the coupling argument is to ensure high intersection between the variables of our polynomial program and the uncorrupted samples. We know that at least a $(1-\epsilon)$-fraction of the samples we observe agree with the uncorrupted samples. To this end, we create indicator variables $w_{i}$, for $i \in[n]$ such that :

$$
\left\{\begin{aligned}
& \sum_{i \in[n]} w_{i} & =(1-\epsilon) n \\
\forall i \in[n] . & w_{i}^{2} & =w_{i} \\
\forall i \in[n] & w_{i}\left(x_{i}^{\prime}-x_{i}\right) & =0 \\
\forall i \in[n] & w_{i}\left(y_{i}^{\prime}-y_{i}\right) & =0
\end{aligned}\right\}
$$

The intersection constraints ensure that our polynomial system variables agree with the observed samples on $(1-\epsilon) n$ points. We note that such constraints are now standard in the literature, and indeed are the only constraints explicitly enforced by [33].

Independence as a Polynomial Inequality. The central challenge in obtaining optimal rates for robust regression is to leverage the independence of the noise and covariates. Since independence is a property of the marginals of $\mathcal{D}$, it is not immediately clear how to leverage it while designing a robust estimator.

However, recall that we do not require independence in full generality and use negatively correlated moments as a proxy for independence. Ideally, we would want to enforce the polynomial inequality corresponding to negatively correlated moments directly on the variables of our polynomial program as follows:

$$
\left\{\begin{aligned}
\forall r \leqslant \frac{k}{2}, & \frac{1}{n} \sum_{i \in[n]}\left(v^{\top} x_{i}^{\prime}\left(y_{i}^{\prime}-\left(x_{i}^{\prime}\right)^{\top} \Theta\right)\right)^{2 r} \\
& \leqslant O(1)\left(\frac{1}{n} \sum_{i \in[n]}\left(v^{\top} x_{i}^{\prime}\right)^{2 r}\right)\left(\frac{1}{n} \sum_{i \in[n]}\left(y_{i}^{\prime}-\left(x_{i}^{\prime}\right)^{\top} \Theta\right)^{2 r}\right)
\end{aligned}\right\}
$$

where $\Theta$ is a variable corresponding to the true hyperplane. To demonstrate feasibility of this constraint, we would require a finite sample analysis, showing that uncorrupted samples from a hypercontractive distribution satisfy the above inequality. Observe, when $r=k / 2$, the LHS is a degree- $k$ polynomial and our distribution may be too heavy-tailed to achieve any concentration.

Instead, we observe that since hypercontractivity is preserved under sampling, we can relax our polynomial constraint by applying hypercontractivity to the terms in the RHS above :

$$
\left\{\begin{aligned}
\forall r \leqslant \frac{k}{2}, & \frac{1}{n} \sum_{i \in[n]}\left(v^{\top} x_{i}^{\prime}\left(y_{i}^{\prime}-\left(x_{i}^{\prime}\right)^{\top} \Theta\right)\right)^{2 r} \\
& \leqslant O(1)\left(\frac{1}{n} \sum_{i \in[n]}\left(v^{\top} x_{i}^{\prime}\right)^{2}\right)^{r}\left(\frac{1}{n} \sum_{i \in[n]}\left(y_{i}^{\prime}-\left(x_{i}^{\prime}\right)^{\top} \Theta\right)^{2}\right)^{r}
\end{aligned}\right\}
$$

In Lemma 5.2 we show that the above inequality is feasible for the uncorrupted samples. In particular, given at least, $d^{\Omega(k)}$ i.i.d. samples from $\mathcal{D}$, the above inequality holds on the samples with high probability.

Perhaps surprisingly, the dependence on $\epsilon$ achieved when $\mathcal{D}$ has negatively correlated moments matches the information theoretically optimal rate for independent noise. We thus expect the notion of negatively correlated moments to lead to new estimators for problems where independence of random variables requires to be formulated as a polynomial inequality.

Hypercontractivity Constraints. Since hypercontractivity is a already a polynomial identity relating the $k$-th moment to the variance of a distribution, it can easily enforced as a constraint. Conveniently, if the underlying distribution $\mathcal{D}$ is hypercontractive, then the uniform distribution over a sufficiently large sample is also sampling also hypercontractive (see Lemma 5.7 in [33]). Therefore, the following constraints are feasible:

$\left\{\begin{array}{lc}\forall r \leqslant \frac{k}{2} & \frac{1}{n} \sum_{i \in[n]}\left\langle x_{i}^{\prime}, v\right\rangle^{2 r} \leqslant\left(\frac{c_{r}}{n} \sum_{i \in[n]}\left\langle x_{i}^{\prime}, v\right\rangle^{2}\right)^{r} \\ \forall r \leqslant \frac{k}{2} & \frac{1}{n} \sum_{i \in[n]}\left(y_{i}^{\prime}-\left\langle\Theta, x_{i}^{\prime}\right\rangle\right)^{2 r} \leqslant\left(\frac{\eta_{r}}{n} \sum_{i \in[n]}\left(y_{i}^{\prime}-\left\langle\Theta, x_{i}^{\prime}\right\rangle\right)^{2}\right)^{r}\end{array}\right\}$

We note that Klivans, Kothari and Meka [33] use hypercontractivity of the uncorrupted samples in their analysis but do not explicitly enforce this as a constraint. Enforcing hypercontractivity explicitly on the samples was used by Kothari and Steurer [36] in the context of robust moment estimation and Kothari and Steinhardt [34] in the context of robustly clustering a mixture of spherical Gaussians.

Gradient Constraints. Finally, it is crucial in our analysis to enforce that the minimizer we are searching for, $\Theta$, has gradient 0 . For the least-squares loss, the gradient has a simple analytic form : for all $v \in \mathbb{R}^{d}$,

$$
\left\{\left\langle v, \frac{1}{n} \sum_{i \in[n]} x_{i}^{\prime}\left(\left\langle x_{i}^{\prime}, \Theta\right\rangle-y_{i}^{\prime}\right)\right\rangle^{k}=0\right\}
$$

While such optimality conditions are often used in the analysis of estimators (as done in [43]), we emphasize that we hardcode the gradient condition into the description of our robust estimator. To the best of our knowledge, no estimator for robust/high-dimensional statistics includes explicit optimality constraints as a part of a polynomial system.

Solving the least-squares objective on the samples subject to the polynomial system described by the aforementioned constraints results in an estimator for robust regression that achieves optimal rate. Recall, this follows immidiately from our robust certifiability proof. However, optimizing polynomial systems can be NP-Hard 
in the worse case, and thus we briefly describe how to avoid this computational intractability next.

\subsection{Efficient Algorithms via Sum-of-Squares}

We use the sum-of-squares method to make the aforementioned polynomial system efficiently computable and provide an outline of this approach (see Section 3 for a formal treatment of sum-ofsquares proofs). Instead of directly solving the polynomial program, let us instead consider finding a distribution, $\mu$, over feasible solutions $w, x^{\prime}, y^{\prime}$ and $\Theta$ that minimizes

$$
\underset{w, x^{\prime}, y^{\prime}, \Theta \sim \mu}{\mathbb{E}}\left[\frac{1}{n} \sum_{i \in[n]}\left(w_{i} y_{i}^{\prime}-\left\langle w_{i} x_{i}^{\prime}, \Theta\right\rangle\right)^{2}\right]
$$

and satisfies the constraints above. Then, it follows from our information theoretic proof (Theorem 4.1) that

$$
\underset{\mu}{\mathbb{E}}\left[\left\|\Sigma^{1 / 2}\left(\Theta^{*}-\Theta\right)\right\|_{2}\right] \leqslant O\left(\epsilon^{1-1 / k}\right) \operatorname{err}_{\mathcal{D}}\left(\Theta^{*}\right)^{1 / 2}
$$

where $\Theta^{*}$ is the optimal hyperplane.

We now face two challenges: finding a distribution over solutions is at least as hard as the original problem and we no longer recover a unique hyperplane. The latter is easy to address by observing that the hyperplane obtained by averaging over the distribution, $\mu$, suffices:

$$
\left\|\Sigma^{1 / 2}\left(\Theta^{*}-\underset{\mu}{\mathbb{E}}[\Theta]\right)\right\|_{2} \leqslant \underset{\mu}{\mathbb{E}}\left[\left\|\Sigma^{1 / 2}\left(\Theta^{*}-\Theta\right)\right\|_{2}\right]
$$

where the inequality follows from convexity of the loss.

Following prior works, it is now natural to instead consider searching for a "pseudo-distribution", $\zeta$, over feasible solutions. A pseudo-distribution is an object similar to a real distribution, but relaxed to allow negative mass on its support (see Subsection 3.1 for a formal treatment). Crucially, a pseudo-distribution over the polynomial program can be computed efficiently by formulating it as a large SDP. To see why this helps, note any polynomial inequality that can be derived using "sum-of-squares" proofs from a set of polynomial constraints using a low-degree sum-of-squares proof remains valid if we replace distributions by "pseudo-distribution".

For instance, if Equation 7 were a polynomial inequality in $w, x^{\prime}, y^{\prime}$ and $\Theta$, obtained by applying simple transformations that admit sum-of-squares proofs, we could replace $\mu$ by $\zeta$, and obtain an efficient estimator. However, Equation 7 is not a polynomial inequality and the proof outlined in Subsection 2.1 is not a low-degree sum-of-squares proof. Therefore, a central technical contribution of our work is to formulate the right polynomial identity bounding the distance between $\Theta^{*}$ and $\Theta$ in terms of the least-squares error incurred by by $\Theta^{*}$, and deriving this bound from the polynomial constraints using a low-degree sum-of-squares proof. We do this in Section 5 .

\subsection{Distribution Families}

We note that our statistical estimator applies to all distributions, $\mathcal{D}$, that are $\left(c_{k}, k\right)$-hypercontractive and the rate is completely determined by whether $\mathcal{D}$ has negatively correlated moments. In particular, for the important special case of heavy-tailed regression with independent noise, we obtain rate proportional to $\epsilon^{1-1 / k}$ for parameter recovery.
However, similar to prior work on hypercontractive distributions, our efficient estimators apply to a more restrictive class, i.e. certifiably hypercontractive distributions (Definition 3.5). Intuitively, this condition captures the criteria that information about degree- $k$ moment upper bounds is "algorithmically accessible". Certifiably hypercontractive distributions are a broad class and include affine transformations of isotropic distributions satisfying Poincaré inequalities and all strongly log-concave distributions. For a detailed discussion of distributions satisfying Poincaré inequalities and their closure properties, we refer the reader to [34, 36].

Surprisingly, while we enforce a constraint corresponding to negatively correlated moments, we do not require a certifiable variant of this condition. Therefore, our efficient estimators hold for regression instances where the true distribution satisfies this condition, including the special case where the noise is independent from the covariates. Finally, our estimators unify various noise models and imply that even in the agnostic setting, the rate degrades only when the noise is positively correlated with the covariates.

\section{PRELIMINARIES}

Throughout this paper, for a vector $v$, we use $\|v\|_{2}$ to denote the Euclidean norm of $v$. For a $n \times m$ matrix $M$, we use $\|M\|_{2}=\max _{\|x\|_{2}=1}$ $\|M x\|_{2}$ to denote the spectral norm of $M$ and $\|M\|_{F}=\sqrt{\sum_{i, j} M_{i, j}^{2}}$ to denote the Frobenius norm of $M$. For symmetric matrices we use $\geq$ to denote the PSD/Loewner ordering over eigenvalues of $M$. Recall, the definition of total variation distance between probability measures:

Definition 3.1 (Total Variation Distance). The TV distance between distributions with PDFs $p, q$ is defined as $\frac{1}{2} \int_{-\infty}^{\infty} \mid p(x)-$ $q(x) \mid d x$.

Given a distribution $\mathcal{D}$ over $\mathbb{R}^{d} \times \mathbb{R}$, we consider the least squares error of a vector $\Theta$ w.r.t. $\mathcal{D}$ to be $\operatorname{err}_{\mathcal{D}}(\Theta)=\mathbb{E}_{x, y \sim \mathcal{D}}\left[(y-\langle x, \Theta\rangle)^{2}\right]$. The linear regression problem minimizes the error over all $\Theta$. The minimizer, $\Theta_{\mathcal{D}}$ of the aformentioned error satisfies the following "gradient condition" : for all $v \in \mathbb{R}^{d}, \mathbb{E}_{x, y \sim \mathcal{D}}\left[\left\langle v, x x^{\top} \Theta_{\mathcal{D}}-x y\right\rangle\right]=$ 0

FACT 3.2 (CONVERgenCE OF EMPIRICAL MOMENTS, IMPlicit IN Lemma 5.5 [36] ). Let $\mathcal{D}$ be a $\left(c_{k}, k\right)$-hypercontractive distribution with covariance $\Sigma$ and let $\mathcal{X}=\left\{x_{1}, \ldots x_{n}\right\}$ be $n=\Omega\left((d \log (d) / \delta)^{k / 2}\right)$ i.i.d. samples from $\mathcal{D}$. Then, with probability at least $1-\delta,(1-0.1) \Sigma \leq$ $\frac{1}{n} \sum_{i}^{n} x_{i} x_{i}^{\top} \leq(1+0.1) \Sigma$.

Fact 3.3 (TV Closeness to Covariance Closeness, Lemma 2.2 [36]). Let $\mathcal{D}_{1}, \mathcal{D}_{2}$ be ( $\left.c_{k}, k\right)$-hypercontractive distributions over $\mathbb{R}^{d}$ such that $\left\|\mathcal{D}-\mathcal{D}^{\prime}\right\|_{T V} \leqslant \epsilon$, where $0<\epsilon<O\left(\left(1 / c_{k}\right)^{\frac{k}{k-1}}\right)$. Let $\Sigma_{1}, \Sigma_{2}$ be the corresponding covariance matrices. Then, for $\delta \leqslant$ $O\left(c_{k} \epsilon^{1-1 / k}\right)<1,(1-\delta) \Sigma_{2} \leq \Sigma_{1} \leq(1+\delta) \Sigma_{2}$.

Lemma 3.4 (Löwner Ordering for Hypercontractive SamPLES). Let $\mathcal{D}$ be a $\left(c_{k}, k\right)$-hypercontractive distribution with covariance $\Sigma$ and and let $\mathcal{U}$ be the uniform distribution over $n$ samples. Then, with probability $1-\delta,\left\|\Sigma^{-1 / 2} \hat{\Sigma} \Sigma^{-1 / 2}-I\right\|_{F} \leqslant \frac{C_{4} d^{2}}{\sqrt{n} \sqrt{\delta}}$, where $\hat{\Sigma}=\frac{1}{n} \sum_{i \in[n]} x_{i} x_{i}^{\top}$. 
Next, we define the technical conditions required for efficient estimators. Formally,

Definition 3.5 (Certifiable Hypercontractivity). A distribution $\mathcal{D}$ on $\mathbb{R}^{d}$ is $\left(c_{k}, k\right)$-certifiably hypercontractive if for all $r \leqslant k / 2$, there exists a degree $O(k)$ sum-of-squares proof (defined below) of the following inequality in the variable $v$

$$
\underset{x \sim \mathcal{D}}{\mathbb{E}}\left[\langle x, v\rangle^{2 r}\right] \leqslant \underset{x \sim \mathcal{D}}{\mathbb{E}}\left[c_{r}\langle x, v\rangle^{2}\right]^{r}
$$

such that $c_{r} \leqslant c_{k}$.

Next, we note that if a distribution $\mathcal{D}$ is certifiably hypercontractive, the uniform distribution over $n$ i.i.d. samples from $\mathcal{D}$ is also certifiably hypercontractive.

Fact 3.6 (Sampling Preserves Certifiable HypercontractivITY, LEMMA 5.5 [36] ). Let $\mathcal{D}$ be a $\left(c_{k}, k\right)$-certifiably hypercontractive distribution on $\mathbb{R}^{d}$. Let $\mathcal{X}$ be a set of $n=\Omega\left((d \log (d / \delta))^{k / 2} / \gamma^{2}\right)$ i.i.d. samples from $\mathcal{D}$. Then, with probability $1-\delta$, the uniform distribution over $\mathcal{X}$ is $\left(c_{k}+\gamma, k\right)$-certifiably hypercontractive.

We also note that certifiably hypercontractivity is preserved under Affine transformations of the distribution.

Fact 3.7 (Certifiable Hypercontractivity under Affine Transformations, Lemma 5.1, 5.2 [36]). Let $x \in \mathbb{R}^{d}$ be a random variable drawn from $a\left(c_{k}, k\right)$-certifiably hypercontractive distribution. Then, for matrix $A$ and vector $b$, the distribution over the random variable $A x+b$ is also $\left(c_{k}, k\right)$-certifiably hypercontractive.

Next, we formally define the condition on the moments and noise that we require to obtain efficient algorithms. We note that for technical reasons it is not simply a polynomial identity encoding Definition 1.5.

Definition 3.8 (Certifiable Negatively Correlated Moments). A distribution $\mathcal{D}$ on $\mathbb{R}^{d} \times \mathbb{R}$ has $O(1)$-certifiable negatively correlated moments if for all $r \leqslant k / 2$ there exists a degree $O(k)$ sum-of-squares proof of the following inequality

$$
\begin{aligned}
& \underset{x, y \sim \mathcal{D}}{\mathbb{E}}\left[\left(v^{\top} x\left(y-x^{\top} \Theta\right)\right)^{2 r}\right] \\
& \leqslant O\left(\lambda_{r}^{r}\right)\left(\mathbb{E}\left[\left(v^{\top} x\right)^{2}\right]^{r}\right)\left(\mathbb{E}\left[\left(y-x^{\top} \Theta\right)^{2}\right]^{r}\right),
\end{aligned}
$$

for a fixed vector $\Theta$.

We defer the preliminaries for sum-of-squares proofs to the full version of this manuscript.

\subsection{SoS Background}

In this subsection, we provide the necessary background for the sum-of-squares proof system. We follow the exposition as it appears in lecture notes by Barak [3], and the preliminary sections of several recent works [1, 2, 31, 32, 36].

Pseudo-Distributions. We can represent a discrete probability distribution over $\mathbb{R}^{n}$ by its probability mass function $D: \mathbb{R}^{n} \rightarrow$ $\mathbb{R}$ such that $D \geqslant 0$ and $\sum_{x \in \operatorname{supp}(D)} D(x)=1$. Similarly, we can describe a pseudo-distribution by its mass function by relaxing the non-negativity constraint, while still passing certain low-degree non-negativity tests.
Definition 3.9 (Pseudo-distribution). A level- $\ell$ pseudo-distribution is a finitely-supported function $D: \mathbb{R}^{n} \rightarrow \mathbb{R}$ such that $\sum_{x} D(x)=1$ and $\sum_{x} D(x) f(x)^{2} \geqslant 0$ for every polynomial $f$ of degree at most $\ell / 2$, where the summation is over all $x$ in the support of $D$.

Next, we define the notion of pseudo-expectation.

Definition 3.10 (Pseudo-expectation). The pseudo-expectation of a function $f$ on $\mathbb{R}^{d}$ with respect to a pseudo-distribution $D$, denoted by $\tilde{\mathbb{E}}_{D(x)}[f(x)]$, is defined as

$$
\tilde{\mathbb{E}}_{D(x)}[f(x)]=\sum_{x} D(x) f(x)
$$

We use the notation $\tilde{\mathbb{E}}_{D(x)}\left[\left(1, x_{1}, x_{2}, \ldots, x_{n}\right)^{\otimes \ell}\right]$ to denote the degree- $\ell$ moment tensor of the pseudo-distribution $D$. In particular, each entry in the moment tensor corresponds to the pseudoexpectation of a monomial of degree at most $\ell$ in $x$. Crucially, there's an efficient separation oracle for moment tensors of pseudodistributions.

FACT $3.11([38,41,42,52])$. For any $n, \ell \in \mathbb{N}$, the following set has a $n^{O(\ell)}$-time weak separation oracle (in the sense of [24]):

$$
\left\{\tilde{\mathbb{E}}_{D(x)}\left(1, x_{1}, x_{2}, \ldots, x_{n}\right)^{\otimes \ell} \mid \text { degree- } \ell \text { pseudo-distribution } D\right\}
$$

This fact, together with the equivalence of weak separation and optimization [24] forms the basis of the sum-of-squares algorithm, as it allows us to efficiently approximately optimize over pseudodistributions.

Given a system of polynomial constraints, denoted by $\mathcal{A}$, we say that it is explicitly bounded if it contains a constraint of the form $\left\{\|x\|^{2} \leqslant M\right\}$. Then, the following fact follows from Fact 3.11 and [24]:

Fact 3.12 (Efficient Optimization over Pseudo-distribuTIONS). There exists an $(n+m)^{O(\ell)}$-time algorithm that, given any explicitly bounded and satisfiable system ${ }^{2} \mathcal{A}$ of $m$ polynomial constraints in $n$ variables, outputs a level- $\ell$ pseudo-distribution that satisfies $\mathcal{A}$ approximately.

We now define basic facts for pseudo-distributions, which extend facts that hold for standard probability distributions, which can be found in several prior works listed above.

Fact 3.13 (CAuchy-Schwarz for Pseudo-distributions). Let $f, g$ be polynomials of degree at most $d$ in indeterminate $x \in \mathbb{R}^{d}$. Then, for any degree d pseudo-distribution $\tilde{\zeta}, \tilde{\mathbb{E}}_{\tilde{\zeta}}[f g] \leqslant \sqrt{\tilde{\mathbb{E}}_{\tilde{\zeta}}\left[f^{2}\right]} \sqrt{\tilde{\mathbb{E}}_{\tilde{\zeta}}\left[g^{2}\right]}$.

Sum-of-squares proofs. Let $f_{1}, f_{2}, \ldots, f_{r}$ and $g$ be multivariate polynomials in $x$. A sum-of-squares proof that the constraints $\left\{f_{1} \geqslant\right.$ $\left.0, \ldots, f_{m} \geqslant 0\right\}$ imply the constraint $\{g \geqslant 0\}$ consists of polynomials $\left(p_{S}\right)_{S \subseteq[m]}$ such that

$$
g=\sum_{S \subseteq[m]} p_{S}^{2} \cdot \Pi_{i \in S} f_{i}
$$

We say that this proof has degree $\ell$ if for every set $S \subseteq[m]$, the polynomial $p_{S}^{2} \Pi_{i \in S} f_{i}$ has degree at most $\ell$. If there is a degree $\ell$ SoS

\footnotetext{
${ }^{2}$ Here, we assume that the bit complexity of the constraints in $\mathcal{A}$ is $(n+m) O(1)$
} 
proof that $\left\{f_{i} \geqslant 0 \mid i \leqslant r\right\}$ implies $\{g \geqslant 0\}$, we write:

$$
\left.\left\{f_{i} \geqslant 0 \mid i \leqslant r\right\}\right|_{\ell}\{g \geqslant 0\}
$$

For all polynomials $f, g: \mathbb{R}^{n} \rightarrow \mathbb{R}$ and for all functions $F: \mathbb{R}^{n} \rightarrow$ $\mathbb{R}^{m}, G: \mathbb{R}^{n} \rightarrow \mathbb{R}^{k}, H: \mathbb{R}^{p} \rightarrow \mathbb{R}^{n}$ such that each of the coordinates of the outputs are polynomials of the inputs, we have the following inference rules.

Low-degree sum-of-squares proofs are sound and complete if we take low-level pseudo-distributions as models. Concretely, sum-ofsquares proofs allow us to deduce properties of pseudo-distributions that satisfy some constraints.

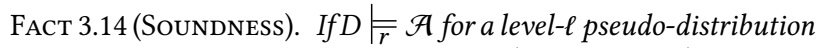
$D$ and there exists a sum-of-squares proof $\left.\mathcal{A}\right|_{r^{\prime}} \mathcal{B}$, then $\left.D\right|_{r \cdot r^{\prime}+r^{\prime}} \mathcal{B}$.

If the pseudo-distribution $D$ satisfies $\mathcal{A}$ only approximately, soundness continues to hold if we require an upper bound on the bitcomplexity of the sum-of-squares $\left.\mathcal{A}\right|_{r^{\prime}} B$ (number of bits required to write down the proof). In our applications, the bit complexity of all sum of squares proofs will be $n^{O(\ell)}$ (assuming that all numbers in the input have bit complexity $\left.n^{O(1)}\right)$. This bound suffices in order to argue about pseudo-distributions that satisfy polynomial constraints approximately.

The following fact shows that every property of low-level pseudodistributions can be derived by low-degree sum-of-squares proofs.

FACt 3.15 (Completeness). Suppose $d \geqslant r^{\prime} \geqslant r$ and $\mathcal{A}$ is a collection of polynomial constraints with degree at most $r$, and $\mathcal{A} \vdash$ $\left\{\sum_{i=1}^{n} x_{i}^{2} \leqslant B\right\}$ for some finite $B$.

Let $\{g \geqslant 0\}$ be a polynomial constraint. If every degree-d pseudodistribution that satisfies $\left.D\right|_{r} \mathcal{A}$ also satisfies $\left.D\right|_{r^{\prime}}\{g \geqslant 0\}$, then for every $\epsilon>0$, there is a sum-of-squares proof $\left.\mathcal{A}\right|_{d}\{g \geqslant-\epsilon\}$.

Basic Sum-of-Squares Proofs. Next, we use the following basic facts regarding sum-of-squares proofs. For further details, we refer the reader to a recent monograph [23].

FACT 3.16 (Operator NORM Bound). Let $A$ be a symmetric $d \times d$ matrix and $v$ be a vector in $\mathbb{R}^{d}$. Then,

$$
\frac{v}{2}\left\{v^{\top} A v \leqslant\|A\|_{2}\|v\|_{2}^{2}\right\} .
$$

Fact 3.17 (SoS Almost Triangle Inequality). Let $f_{1}, f_{2}, \ldots, f_{r}$ be indeterminates. Then,

$$
\frac{f_{1}, f_{2}, \ldots, f_{r}}{2 t}\left\{\left(\sum_{i \leqslant r} f_{i}\right)^{2 t} \leqslant r^{2 t-1}\left(\sum_{i=1}^{r} f_{i}^{2 t}\right)\right\} .
$$

FACT 3.18 (SoS HöldeR's Inequality). Let $w_{1}, \ldots w_{n}$ be indeterminates and let $f_{1}, \ldots f_{n}$ be polynomials of degree $m$ in vector valued variable $x$. Let $k$ be a power of 2 . Then,

$$
\begin{aligned}
&\left\{w_{i}^{2}=w_{i}, \forall i \in[n]\right\} \mid \frac{x, w}{2 k m} \\
&\left\{\left(\frac{1}{n} \sum_{i=1}^{n} w_{i} f_{i}\right)^{k} \leqslant\left(\frac{1}{n} \sum_{i=1}^{n} w_{i}\right)^{k-1}\left(\frac{1}{n} \sum_{i=1}^{n} f_{i}^{k}\right)\right\} .
\end{aligned}
$$

We also use the following fact that allows us to cancel powers of indeterminates within the sum-of-squares proof system.
Fact 3.19 (CAncellation Within SoS, Lemma 9.4 [2] ). Let $a, C$ be indeterminates. Then,

$$
\{a \geqslant 0\} \cup\left\{a^{2 t} \leqslant C a^{t}\right\} \mid \frac{a, C}{2 t}\left\{a^{2 t} \leqslant C^{2}\right\} .
$$

\section{ROBUST CERTIFIABILITY AND INFORMATION THEORETIC ESTIMATORS}

In this section, we provide an estimator that obtains the information theoretically optimal rate for robust regression. We note that we consider the setting where both the covariates and the noise are hypercontractive and the are independent of each other. This setting displays all the key ideas of our estimator. Further, our estimator extends to the remaining settings, such as bounded dependent noise, by simple modifications to the subsequent analysis.

Theorem 4.1 (Robust Certifiability with Optimal Rate). Given $\epsilon>0$, let $\mathcal{D}, \mathcal{D}^{\prime}$ be distributions over $\mathbb{R}^{d} \times \mathbb{R}$ such that the respective marginal distributions over $\mathbb{R}^{d}$, denoted by $\mathcal{D}_{X}, \mathcal{D}_{X}^{\prime}$, are $\left(c_{k}, k\right)$-hypercontractive and $\left\|\mathcal{D}-\mathcal{D}^{\prime}\right\|_{T V} \leqslant \epsilon$. Let $\mathcal{R}_{\mathcal{D}}\left(\epsilon, \Sigma_{\mathcal{D}}, \Theta_{\mathcal{D}}\right)$ and $\mathcal{R}_{\mathcal{D}^{\prime}}\left(\epsilon, \Sigma_{\mathcal{D}^{\prime}}, \Theta_{\mathcal{D}^{\prime}}\right)$ be the corresponding instances of robust regression such that $\mathcal{D}, \mathcal{D}^{\prime}$ have negatively correlated moments. Further, for $(x, y) \sim \mathcal{D}, \mathcal{D}^{\prime}$, let the marginal distribution over $y-$ $\left\langle x, \mathbb{E}\left[x x^{\top}\right]^{-1} \mathbb{E}[x y]\right\rangle$ be $\left(\eta_{k}, k\right)$-hypercontractive Then,

$$
\begin{aligned}
\left\|\Sigma_{\mathcal{D}}^{1 / 2}\left(\Theta_{\mathcal{D}}-\Theta_{\mathcal{D}^{\prime}}\right)\right\|_{2} \leqslant & \mathcal{O}\left(\sqrt{c_{k} \eta_{k}} \epsilon^{1-1 / k}\right) \\
& \left(\operatorname{err}_{\mathcal{D}}\left(\Theta_{\mathcal{D}}\right)^{1 / 2}+\operatorname{err}_{\mathcal{D}^{\prime}}\left(\Theta_{\mathcal{D}^{\prime}}\right)^{1 / 2}\right)
\end{aligned}
$$

Further,

$$
\begin{aligned}
\operatorname{err}_{\mathcal{D}}\left(\Theta_{\mathcal{D}^{\prime}}\right) \leqslant & \left(1+O\left(c_{k} \eta_{k} \epsilon^{2-2 / k}\right)\right) \operatorname{err}_{\mathcal{D}}\left(\Theta_{\mathcal{D}}\right) \\
& +O\left(c_{k} \eta_{k} \epsilon^{2-2 / k}\right) \operatorname{err}_{\mathcal{D}^{\prime}}\left(\Theta_{\mathcal{D}^{\prime}}\right)
\end{aligned}
$$

Proof. Consider a maximal coupling of $\mathcal{D}, \mathcal{D}^{\prime}$ over $(x, y) \times$ $\left(x^{\prime}, y^{\prime}\right)$, denoted by $\mathcal{G}$, such that the marginal of $\mathcal{G}(x, y)$ is $\mathcal{D}$, the marginal on $\left(x^{\prime}, y^{\prime}\right)$ is $\mathcal{D}^{\prime}$ and $\mathbb{P}_{\mathcal{G}}\left[\mathbb{I}\left\{(x, y)=\left(x^{\prime}, y^{\prime}\right)\right\}\right]=1-\epsilon$. Then, for all $v$,

$$
\begin{aligned}
&\left\langle v, \Sigma_{\mathcal{D}}\left(\Theta_{\mathcal{D}}-\Theta_{\mathcal{D}^{\prime}}\right)\right\rangle= \underset{\mathcal{G}}{\mathbb{E}}\left[\left\langle v, x x^{\top}\left(\Theta_{\mathcal{D}}-\Theta_{\mathcal{D}^{\prime}}\right)+x y-x y\right\rangle\right] \\
&=\underset{\mathcal{G}}{\mathbb{E}}\left[\left\langle v, x\left(\left\langle x, \Theta_{\mathcal{D}}\right\rangle-y\right)\right\rangle\right] \\
&+\underset{\mathcal{G}}{\mathbb{E}}\left[\left\langle v, x\left(y-\left\langle x, \Theta_{\mathcal{D}^{\prime}}\right\rangle\right)\right\rangle\right]
\end{aligned}
$$

Since $\Theta_{\mathcal{D}}$ is the minimizer for the least squares loss, we have the following gradient condition : for all $v \in \mathbb{R}^{d}$,

$$
\underset{(x, y) \sim \mathcal{D}}{\mathbb{E}}\left[\left\langle v,\left(\left\langle x, \Theta_{\mathcal{D}}\right\rangle-y\right) x\right\rangle\right]=0
$$

Since $\mathcal{G}$ is a coupling, using the gradient condition (13) and using that $1=\mathbb{I}\left\{(x, y)=\left(x^{\prime}, y^{\prime}\right)\right\}+\mathbb{I}\left\{(x, y) \neq\left(x^{\prime}, y^{\prime}\right)\right\}$, we can rewrite equation (12) as 


$$
\begin{aligned}
\left\langle v, \Sigma\left(\Theta_{\mathcal{D}}-\Theta_{\mathcal{D}^{\prime}}\right)\right\rangle= & \underset{\mathcal{G}}{\mathbb{E}}\left[\left\langle v, x\left(y-\left\langle x, \Theta_{\mathcal{D}^{\prime}}\right\rangle\right)\right\rangle \mathbb{I}\left\{(x, y)=\left(x^{\prime}, y^{\prime}\right)\right\}\right] \\
& +\underset{\mathcal{G}}{\mathbb{E}}\left[\left\langle v, x\left(y-\left\langle x, \Theta_{\mathcal{D}^{\prime}}\right\rangle\right)\right\rangle \mathbb{I}\left\{(x, y) \neq\left(x^{\prime}, y^{\prime}\right)\right\}\right] \\
= & \underset{\mathcal{G}}{\mathbb{E}}\left[\left\langle v, x^{\prime}\left(y^{\prime}-\left\langle x^{\prime}, \Theta_{\mathcal{D}^{\prime}}\right\rangle\right)\right\rangle \mathbb{I}\left\{(x, y)=\left(x^{\prime}, y^{\prime}\right)\right\}\right] \\
& +\underset{\mathcal{G}}{\mathbb{E}}\left[\left\langle v, x\left(y-\left\langle x, \Theta_{\mathcal{D}^{\prime}}\right\rangle\right)\right\rangle \mathbb{I}\left\{(x, y) \neq\left(x^{\prime}, y^{\prime}\right)\right\}\right]
\end{aligned}
$$

Consider the first term in the last equality above. Using the gradient condition for $\Theta_{\mathcal{D}^{\prime}}$ along with Hölder's Inequality, we have

$$
\begin{aligned}
\left|\mathbb{E}_{\mathcal{G}}\left[\left\langle v, x^{\prime}\left(y^{\prime}-\left\langle x^{\prime}, \Theta_{\mathcal{D}^{\prime}}\right\rangle\right)\right\rangle \mathbb{I}\left\{(x, y)=\left(x^{\prime}, y^{\prime}\right)\right\}\right]\right| \\
=\left|\underset{\mathcal{G}}{\mathbb{E}}\left[\left\langle v, x^{\prime}\left(y^{\prime}-\left\langle x^{\prime}, \Theta_{\mathcal{D}^{\prime}}\right\rangle\right)\right\rangle \mathbb{I}\left\{(x, y) \neq\left(x^{\prime}, y^{\prime}\right)\right\}\right]\right| \\
\leqslant\left|\underset{\mathcal{G}}{\mathbb{E}}\left[\mathbb{I}\left\{(x, y) \neq\left(x^{\prime}, y^{\prime}\right)\right\}^{k /(k-1)}\right]^{(k-1) / k}\right| \\
\cdot\left|\underset{\mathcal{D}^{\prime}}{\mathbb{E}}\left[\left\langle v, x^{\prime}\left(y^{\prime}-\left\langle x^{\prime}, \Theta_{\mathcal{D}^{\prime}}\right\rangle\right)\right\rangle^{k}\right]^{1 / k}\right|
\end{aligned}
$$

Observe, since $\mathcal{G}$ is a maximal coupling, the first term above is bounded by $\epsilon^{1-1 / k}$. Further, since $\mathcal{D}^{\prime}$ has negatively correlated moments,

$$
\begin{aligned}
& \underset{\mathcal{D}^{\prime}}{\mathbb{E}}\left[\left\langle v, x^{\prime}\right\rangle^{k} \cdot\left(y^{\prime}-\left\langle x^{\prime}, \Theta_{\mathcal{D}^{\prime}}\right\rangle\right)^{k}\right] \\
& =\underset{\mathcal{D}^{\prime}}{\mathbb{E}}\left[\left\langle v, x^{\prime}\right\rangle^{k}\right] \underset{\mathcal{D}^{\prime}}{\mathbb{E}}\left[\left(y^{\prime}-\left\langle x^{\prime}, \Theta_{\mathcal{D}^{\prime}}\right\rangle\right)^{k}\right]
\end{aligned}
$$

By hypercontractivity of the covariates and the noise, we have

$$
\begin{aligned}
& \underset{\mathcal{D}^{\prime}}{\mathbb{E}}\left[\left\langle v, x^{\prime}\right\rangle^{k}\right]^{1 / k} \underset{\mathcal{D}^{\prime}}{\mathbb{E}}\left[\left(y^{\prime}-\left\langle x^{\prime}, \Theta_{\mathcal{D}^{\prime}}\right\rangle\right)^{k}\right]^{1 / k} \\
& \leqslant O\left(\sqrt{c_{k} \eta_{k}}\right)\left(v^{\top} \Sigma_{\mathcal{D}^{\prime}} v\right)^{1 / 2} \underset{x^{\prime}, y^{\prime} \sim \mathcal{D}^{\prime}}{\mathbb{E}}\left[\left(y^{\prime}-\left\langle x^{\prime}, \Theta_{\mathcal{D}^{\prime}}\right\rangle\right)^{2}\right]^{1 / 2}
\end{aligned}
$$

Therefore, we can restate (15) as follows

$$
\begin{aligned}
& \left|\underset{\mathcal{G}}{\mathbb{E}}\left[\left\langle v, x^{\prime}\left(y^{\prime}-\left\langle x^{\prime}, \Theta_{\mathcal{D}^{\prime}}\right\rangle\right)\right\rangle \mathbb{I}\left\{(x, y)=\left(x^{\prime}, y^{\prime}\right)\right\}\right]\right| \\
& \leqslant O\left(\sqrt{c_{k} \eta_{k}} \epsilon^{\frac{k-1}{k}}\right)\left(v^{\top} \Sigma_{\mathcal{D}^{\prime}} v\right)^{\frac{1}{2}} \underset{x^{\prime}, y^{\prime} \sim \mathcal{D}^{\prime}}{\mathbb{E}}\left[\left(y^{\prime}-\left\langle x^{\prime}, \Theta_{\mathcal{D}^{\prime}}\right\rangle\right)^{2}\right]^{\frac{1}{2}}
\end{aligned}
$$

It remains to bound the second term in the last equality of equation (14), and we proceed as follows :

$$
\begin{aligned}
\underset{\mathcal{G}}{\mathbb{E}}\left[\left\langle v, x\left(y-\left\langle x, \Theta_{\mathcal{D}^{\prime}}\right\rangle\right)\right\rangle \mathbb{I}\left\{(x, y) \neq\left(x^{\prime}, y^{\prime}\right)\right\}\right] \\
=\underset{\mathcal{G}}{\mathbb{E}}\left[\left\langle v, x x^{\top}\left(\Theta_{\mathcal{D}}-\Theta_{\mathcal{D}^{\prime}}\right)\right\rangle \mathbb{I}\left\{(x, y) \neq\left(x^{\prime}, y^{\prime}\right)\right\}\right] \\
\quad+\underset{\mathcal{G}}{\mathbb{E}}\left[\left\langle v, x\left(y-\left\langle x, \Theta_{\mathcal{D}}\right\rangle\right)\right\rangle \mathbb{I}\left\{(x, y) \neq\left(x^{\prime}, y^{\prime}\right)\right\}\right]
\end{aligned}
$$

We bound the two terms above separately. Observe, by applying Hölder's Inequality to the first term, we have

$$
\begin{aligned}
& \underset{\mathcal{G}}{\mathbb{E}}\left[\left\langle v, x x^{\top}\left(\Theta_{\mathcal{D}}-\Theta_{\mathcal{D}^{\prime}}\right)\right\rangle \mathbb{I}\left\{(x, y) \neq\left(x^{\prime}, y^{\prime}\right)\right\}\right] \\
& \leqslant \underset{\mathcal{G}}{\mathbb{E}}\left[\mathbb{I}\left\{(x, y) \neq\left(x^{\prime}, y^{\prime}\right)\right\}\right]^{\frac{k-2}{k}} \underset{\mathcal{G}}{\mathbb{E}}\left[\left\langle v, x x^{\top}\left(\Theta_{\mathcal{D}}-\Theta_{\mathcal{D}^{\prime}}\right)\right\rangle^{\frac{k}{2}}\right]^{\frac{2}{k}} \\
& \leqslant \epsilon^{\frac{k-2}{k}} \underset{\mathcal{G}}{\mathbb{E}}\left[\left\langle v, x x^{\top}\left(\Theta_{\mathcal{D}}-\Theta_{\mathcal{D}^{\prime}}\right)\right\rangle^{\frac{k}{2}}\right]^{\frac{2}{k}}
\end{aligned}
$$

To bound the second term in equation 17, we again use Hölder's Inequality followed $\mathcal{D}$ having negatively correlated moments,

$$
\begin{aligned}
& \underset{\mathcal{G}}{\mathbb{E}}\left[\left\langle v, x\left(y-\left\langle x, \Theta_{\mathcal{D}}\right\rangle\right)\right\rangle \mathbb{I}\left\{(x, y) \neq\left(x^{\prime}, y^{\prime}\right)\right\}\right] \\
& \leqslant \underset{\mathcal{G}}{\mathbb{E}}\left[\mathbb{I}\left\{(x, y) \neq\left(x^{\prime}, y^{\prime}\right)\right\}\right]^{\frac{k-1}{k}} \underset{\mathcal{G}}{\mathbb{E}}\left[\left\langle v, x\left(y-\left\langle x, \Theta_{\mathcal{D}}\right\rangle\right)\right\rangle^{k}\right]^{\frac{1}{k}} \\
& \leqslant \epsilon^{\frac{k-1}{k}} \underset{x \sim \mathcal{D}}{\mathbb{E}}\left[\langle v, x\rangle^{k}\right]^{1 / k} \underset{x, y \sim \mathcal{D}}{\mathbb{E}}\left[\left(y-\left\langle x, \Theta_{\mathcal{D}}\right\rangle\right)^{k}\right]^{1 / k} \\
& \leqslant \epsilon^{\frac{k-1}{k}} \sqrt{c_{k} \eta_{k}}\left(v^{\top} \Sigma_{\mathcal{D}} v\right)^{1 / 2} \underset{x, y \sim \mathcal{D}}{\mathbb{E}}\left[\left(y-\left\langle x, \Theta_{\mathcal{D}}\right\rangle\right)^{2}\right]^{1 / 2}
\end{aligned}
$$

where the last inequality follows from hypercontractivity of the covariates and noise. Substituting the upper bounds obtained in Equations (18) and (19) back in to (17),

$$
\begin{aligned}
\underset{\mathcal{G}}{\mathbb{E}} & {\left[\left\langle v, x\left(y-\left\langle x, \Theta_{\mathcal{D}^{\prime}}\right\rangle\right)\right\rangle \mathbb{I}\left\{(x, y) \neq\left(x^{\prime}, y^{\prime}\right)\right\}\right] } \\
\leqslant & \epsilon^{\frac{k-2}{k}} \underset{\mathcal{G}}{\mathbb{E}}\left[\left\langle v, x x^{\top}\left(\Theta_{\mathcal{D}}-\Theta_{\mathcal{D}^{\prime}}\right)\right\rangle^{\frac{k}{2}}\right]^{\frac{2}{k}} \\
& +\epsilon^{\frac{k-1}{k}} \sqrt{c_{k} \eta_{k}}\left(v^{\top} \Sigma_{\mathcal{D}^{v}}\right)^{1 / 2} \underset{x, y \sim \mathcal{D}}{\mathbb{E}}\left[\left(y-\left\langle x, \Theta_{\mathcal{D}}\right\rangle\right)^{2}\right]^{1 / 2}
\end{aligned}
$$

Therefore, we can now upper bound both terms in Equation (14) as follows:

$$
\begin{aligned}
\left\langle v, \Sigma_{\mathcal{D}}\left(\Theta_{\mathcal{D}}-\Theta_{\mathcal{D}^{\prime}}\right)\right\rangle & \\
\leqslant & O\left(c_{k} \eta_{k} \epsilon^{\frac{k-1}{k}}\right)\left(v^{\top} \Sigma_{\mathcal{D}^{\prime}} v\right)^{1 / 2} \underset{x^{\prime}, y^{\prime} \sim \mathcal{D}^{\prime}}{\mathbb{E}}\left[\left(y^{\prime}-\left\langle x^{\prime}, \Theta_{\mathcal{D}^{\prime}}\right\rangle\right)^{2}\right]^{1 / 2} \\
& +O\left(\epsilon^{\frac{k-2}{k}}\right) \underset{\mathcal{G}}{\mathbb{E}}\left[\left\langle v, x x^{\top}\left(\Theta_{\mathcal{D}}-\Theta_{\mathcal{D}^{\prime}}\right)\right\rangle^{k / 2}\right]^{2 / k} \\
& +O\left(\epsilon^{\frac{k-1}{k}} \sqrt{c_{k} \eta_{k}}\right)\left(v^{\top} \Sigma_{\mathcal{D}^{v}}\right)^{1 / 2} \underset{x, y \sim \mathcal{D}}{\mathbb{E}}\left[\left(y-\left\langle x, \Theta_{\mathcal{D}}\right\rangle\right)^{2}\right]^{1 / 2}
\end{aligned}
$$

Recall, since the marginals of $\mathcal{D}$ and $\mathcal{D}^{\prime}$ on $\mathbb{R}^{d}$ are $\left(c_{k}, k\right)$ - hypercontractive and $\left\|\mathcal{D}-\mathcal{D}^{\prime}\right\|_{\mathrm{TV}} \leqslant \epsilon$, it follows from Fact 3.3 that

$$
(1-0.1) \Sigma_{\mathcal{D}^{\prime}} \leq \Sigma_{\mathcal{D}} \leq(1+0.1) \Sigma_{\mathcal{D}^{\prime}}
$$

when $\epsilon \leqslant O\left(\left(1 / c_{k} k\right)^{k / k-1}\right)$. Now, consider the substitution $v=$ $\Theta_{\mathcal{D}}-\Theta_{\mathcal{D}^{\prime}}$. Observe,

$$
\begin{aligned}
\underset{\mathcal{G}}{\mathbb{E}}\left[\left\langle v, x x^{\top}\left(\Theta_{\mathcal{D}}-\Theta_{\mathcal{D}^{\prime}}\right)\right\rangle^{k / 2}\right]^{2 / k} & =\underset{\mathcal{D}}{\mathbb{E}}\left[\left\langle x,\left(\Theta_{\mathcal{D}}-\Theta_{\mathcal{D}^{\prime}}\right)\right\rangle^{k}\right]^{2 / k} \\
& \leqslant c_{k}\left\|\Sigma_{\mathcal{D}}^{1 / 2}\left(\Theta_{\mathcal{D}}-\Theta_{\mathcal{D}^{\prime}}\right)\right\|_{2}^{2}
\end{aligned}
$$


Then, using the bounds in (21) and (22) along with $v=\Theta_{\mathcal{D}}-\Theta_{\mathcal{D}^{\prime}}$ in Equation 20, we have

$$
\begin{aligned}
& \left(1-O\left(\epsilon^{\frac{k-2}{k}} c_{k}\right)\right)\left\|\Sigma_{\mathcal{D}}^{1 / 2}\left(\Theta_{\mathcal{D}}-\Theta_{\mathcal{D}^{\prime}}\right)\right\|_{2}^{2} \\
& \leqslant O\left(\sqrt{c_{k} \eta_{k}} \epsilon^{\frac{k-1}{k}}\right)\left\|\Sigma_{\mathcal{D}}^{1 / 2}\left(\Theta_{\mathcal{D}}-\Theta_{\mathcal{D}^{\prime}}\right)\right\|_{2} \cdot \\
& \quad\left(\underset{x^{\prime}, y^{\prime} \sim \mathcal{D}^{\prime}}{\mathbb{E}}\left[\left(y^{\prime}-\left\langle x^{\prime}, \Theta_{\mathcal{D}^{\prime}}\right\rangle\right)^{2}\right]^{\frac{1}{2}}+\underset{x, y \sim \mathcal{D}}{\mathbb{E}}\left[\left(y-\left\langle x, \Theta_{\mathcal{D}}\right\rangle\right)^{2}\right]^{\frac{1}{2}}\right)
\end{aligned}
$$

Dividing out (23) by $\left(1-O\left(\epsilon^{\frac{k-2}{k}} c_{k}\right)\right)\left\|\Sigma_{\mathcal{D}}^{1 / 2}\left(\Theta_{\mathcal{D}}-\Theta_{\mathcal{D}^{\prime}}\right)\right\|_{2}^{2}$ and observing that $O\left(\epsilon^{\frac{k-2}{k}} c_{k}\right)$ is upper bounded by a fixed constant less than 1 yields the parameter recovery bound.

Given the parameter recovery result above, we bound the leastsquares loss between the two hyperplanes on $\mathcal{D}$ as follows:

$$
\begin{aligned}
& \left|\operatorname{err}_{\mathcal{D}}\left(\Theta_{\mathcal{D}}\right)-\operatorname{err}_{\mathcal{D}}\left(\Theta_{\mathcal{D}^{\prime}}\right)\right| \\
& =\left|\underset{(x, y) \sim \mathcal{D}}{\mathbb{E}}\left[\left\langle x,\left(\Theta_{\mathcal{D}}-\Theta_{\mathcal{D}^{\prime}}\right)\right\rangle^{2}+2\left(y-x^{\top} \Theta_{\mathcal{D}}\right) x^{\top}\left(\Theta_{\mathcal{D}}-\Theta_{\mathcal{D}^{\prime}}\right)\right]\right| \\
& \leqslant \\
& \quad\left(c_{k} \eta_{k} \epsilon^{2-2 / k}\right) . \\
& \quad\left(\underset{x^{\prime}, y^{\prime} \sim \mathcal{D}^{\prime}}{\mathbb{E}}\left[\left(y^{\prime}-\left\langle x^{\prime}, \Theta_{\mathcal{D}^{\prime}}\right\rangle\right)^{2}\right]+\underset{x, y \sim \mathcal{D}}{\mathbb{E}}\left[\left(y-\left\langle x, \Theta_{\mathcal{D}}\right\rangle\right)^{2}\right]\right)
\end{aligned}
$$

where the last inequality follows from observing the gradient condition and squaring the parameter recovery bound.

Next, we consider the setting where the noise is allowed to dependent arbitrarily on the covariates, which captures the well-studied agnostic model. With a slightly modification in our certifiability proof above (using Cauchy-Schwarz instead of independence), we obtain the optimal rate in this setting. We defer the details to the full version.

Corollary 4.2 (Robust Regression with Dependent Noise). Let $\mathcal{D}, \mathcal{D}^{\prime}$ be distributions over $\mathbb{R}^{d} \times \mathbb{R}$ and let $\mathcal{R}_{\mathcal{D}}\left(\epsilon, \Sigma_{\mathcal{D}}, \Theta_{\mathcal{D}}\right)$, $\mathcal{R}_{\mathcal{D}^{\prime}}\left(\epsilon, \Sigma_{\mathcal{D}^{\prime}}, \Theta_{\mathcal{D}^{\prime}}\right)$ be robust regression instances satisfying the hypothesis in Theorem 4.1 such that the negatively correlated moments condition is not satisfied. Then,

$$
\begin{aligned}
& \left\|\Sigma_{\mathcal{D}}^{1 / 2}\left(\Theta_{\mathcal{D}}-\Theta_{\mathcal{D}^{\prime}}\right)\right\|_{2} \\
& \leqslant O\left(\sqrt{c_{k} \eta_{k}} \epsilon^{1-2 / k}\right)\left(\operatorname{err}_{\mathcal{D}}\left(\Theta_{\mathcal{D}}\right)^{1 / 2}+\operatorname{err}_{\mathcal{D}^{\prime}}\left(\Theta_{\mathcal{D}^{\prime}}\right)^{1 / 2}\right) .
\end{aligned}
$$

\section{ROBUST REGRESSION IN POLYNOMIAL TIME}

In this section, we describe an algorithm to compute our robust estimator for linear regression efficiently. We consider a polynomial system that encodes our robust estimator. We then consider a sum-of-squares relaxation of this program and compute an approximately optimal solution for our relaxation. To analyze our algorithm, we consider the dual of the sum-of-squares relaxation and show that the sum-of-squares proof system caputures a variant of our robust identifiability proof. We note that given space constraints, we defer all proofs of intermediate lemmas to the full version.
We begin by recalling notation: let $\mathcal{D}$ be a distribution over $\mathbb{R}^{d} \times \mathbb{R}$ such that it is $\left(\lambda_{k}, k\right)$-certifiably hypercontractive. Let $\mathcal{X}=\left\{\left(x_{1}^{*}, y_{1}^{*}\right),\left(x_{2}^{*}, y_{2}^{*}\right) \ldots\left(x_{n}^{*}, y_{n}^{*}\right)\right\}$ denote $n$ uncorrupted i.i.d samples from $\mathcal{D}$ and let $\mathcal{X}_{\epsilon}=.\left\{\left(x_{1}, y_{1}\right),\left(x_{2}, y_{2}\right) \ldots\left(x_{n}, y_{n}\right)\right\}$ be an $\epsilon-$ corruption of the samples $\mathcal{X}$, drawn from a Robust Regression model, $\mathcal{R}_{\mathcal{D}}\left(\epsilon, \Sigma^{*}, \Theta^{*}\right)$ (Model 1.1). We consider a polynomial system in the variables $\mathcal{X}^{\prime}=\left\{\left(x_{1}^{\prime}, y_{1}^{\prime}\right),\left(x_{2}^{\prime}, y_{2}^{\prime}\right) \ldots\left(x_{n}^{\prime}, y_{n}^{\prime}\right)\right\}$ and $w_{i} \in\{0,1\}$ as shown in Table 2.

We show that optimizing an appropriate convex function subject to the aforementioned constraint system results in an efficiently computable robust estimator for regression, achieving the information-theoretically optimal rate. Formally,

Theorem 5.1 (Robust Regression with Negatively CorreLAted Moments, Theorem 1.7 Restated). Given $k \in \mathbb{N}, \epsilon>0$ and $n \geqslant n_{0}$ samples $\mathcal{X}_{\epsilon}=\left\{\left(x_{1}, y_{1}\right), \ldots\left(x_{n}, y_{n}\right)\right\}$ from $\mathcal{R}_{\mathcal{D}}\left(\epsilon, \Sigma^{*}, \Theta^{*}\right)$, where $\mathcal{D}$ is a $\left(\lambda_{k}, k\right)$-certifiably hypercontractive distribution over $\mathbb{R}^{d} \times \mathbb{R}$. Further, $\mathcal{D}$ has certifiable negatively correlated moments. Then, Algorithm 5.6 runs in $n^{O(k)}$ time and outputs an estimator $\tilde{\mathbb{E}}_{\tilde{\zeta}}[\Theta]$ such that when $n_{0}=\Omega\left((d \log (d))^{\Omega(k)} / \gamma^{2}\right)$ with probability $1-1 / \operatorname{poly}(d)$ (over the draw of the input),

$$
\left\|\left(\Sigma^{*}\right)^{1 / 2}\left(\Theta^{*}-\tilde{\mathbb{E}}_{\tilde{\zeta}}[\Theta]\right)\right\|_{2} \leqslant O\left(\lambda_{k} \epsilon^{1-1 / k}+\lambda_{k} \gamma\right) \operatorname{err}_{\mathcal{D}}\left(\Theta^{*}\right)^{1 / 2}
$$

Further,

$$
\operatorname{err}_{\mathcal{D}}\left(\tilde{\mathbb{E}}_{\tilde{\zeta}}[\Theta]\right) \leqslant\left(1+O\left(\lambda_{k}^{2} \epsilon^{2-2 / k}+\lambda_{k}^{2} \gamma^{2}\right)\right) \operatorname{err}_{\mathcal{D}}\left(\Theta^{*}\right) .
$$

Efficient Estimator for Arbitrary Noise. We note that an argument similar to the one presented for Theorem 5.1 results in a polynomial time estimator when the regression instance does not have negatively correlated moments (definition 1.5), albeit at a slightly worse rate. At a high level, we simply do not enforce the negatively correlated moments constraint in our polynomial system $\mathcal{A}_{\epsilon, \lambda_{k}}$ and instead use the SoS Cauchy-Schwarz inequality in our key technical lemma (Lemma 5.3).

\subsection{Analysis}

We begin by observing that we can efficiently optimize the polynomial program above since it admits a compact representation. In particular, $\mathcal{A}_{\epsilon, \lambda_{k}}$ can be represented as a system of $\operatorname{poly}\left(n^{k}\right)$ constraints in $n^{O(k)}$ variables. We refer the reader to [23] for a detailed overview on how to efficiently implement the aforementioned constraints.

Lemma 5.2 (Soundness of the Constraint System). Given $n \geqslant$ $n_{0}$ samples from $\mathcal{R}_{\mathcal{D}}\left(\epsilon, \Theta^{*}, \Sigma\right)$, with probability at least $1-1 /$ poly $(d)$ over the draw of the samples, there exists an assignment for $w, x^{\prime}, y^{\prime}$ and $\Theta$ such that $\mathcal{A}_{\epsilon, \lambda_{k}}$ is feasible when $n_{0}=\left((d \log (d))^{\Omega(k)}\right)$.

Let $\hat{\Sigma}$ be the empirical covariance of the uncorrupted samples $X$ and let $\hat{\Theta}$ be an optimizer for the empirical loss. Applying Theorem 4.1 with $\mathcal{D}$ being the uniform distribution on the uncorrupted samples $\mathcal{X}$ and $\mathcal{D}^{\prime}$ being the uniform distribution on $x_{i}^{\prime}$, we get

$$
\left\|\hat{\Sigma}^{1 / 2}(\Theta-\hat{\Theta})\right\|_{2} \leqslant O\left(\lambda_{k} \epsilon^{1-1 / k}\right) \operatorname{err}_{\mathcal{D}}\left(\Theta^{*}\right)^{1 / 2}
$$

Observe, the aforementioned bound is not a polynomial identity and thus cannot be expressed in the SoS framework. Therefore, we 
Table 2: Constraint System

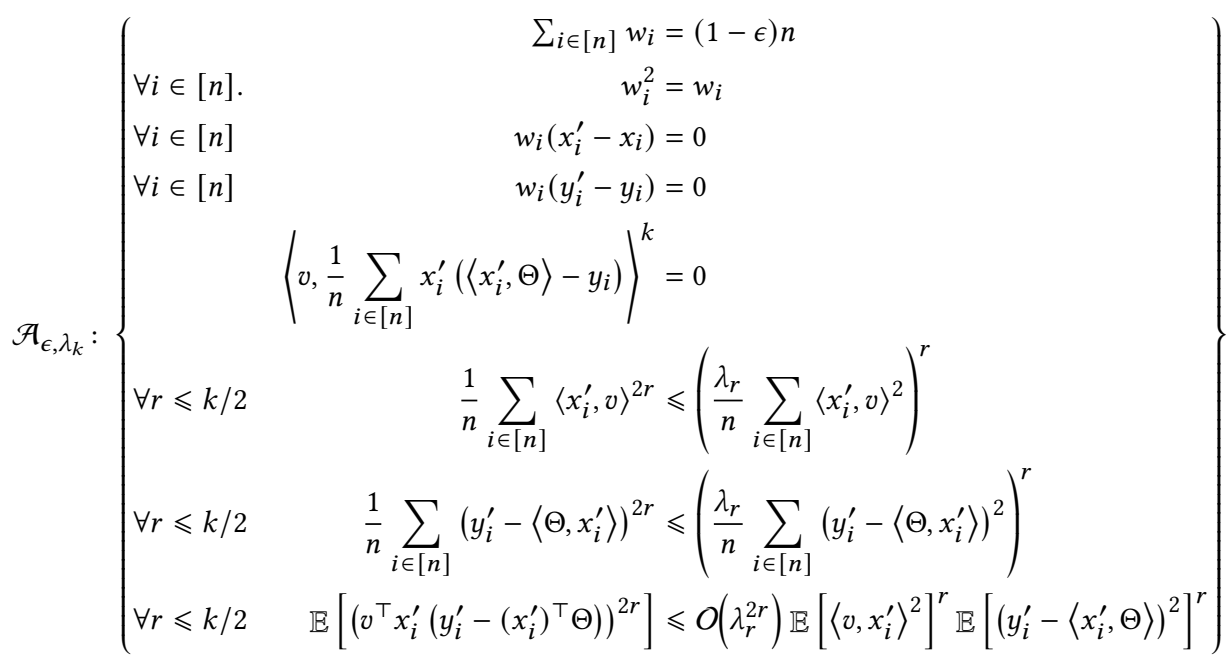

provide a low-degree SoS proof of a slightly modified version of the inequality above, that is inspired by our information theoretic identifiability proof in Theorem 4.1.

Lemma 5.3 (Robust Identifiability in SoS). Consider the hypothesis of Theorem 5.1. Let $w, x^{\prime}, y^{\prime}$ and $\Theta$ be feasible solutions for the polynomial constraint system $\mathcal{A}$. Let $\hat{\Theta}=\arg \min _{\Theta} \frac{1}{n} \sum_{i \in[n]}\left(y_{i}^{*}-\right.$ $\left.\left\langle x_{i}^{*}, \Theta\right\rangle\right)^{2}$ be the empirical loss minimizer on the uncorrupted samples and let $\hat{\Sigma}=\mathbb{E}\left[x_{i}^{*}\left(x_{i}^{*}\right)^{\top}\right]$ be the covariance of the uncorrupted samples. Then,

$$
\begin{aligned}
\mathcal{A} \vdash\{ & \left\|\hat{\Sigma}^{1 / 2}(\hat{\Theta}-\Theta)\right\|_{2}^{2 k} \\
& \leqslant 2^{3 k}(2 \epsilon)^{k-1} \lambda_{k}^{k} \sigma^{k / 2}\left\|\mathbb{E}\left[x_{i}^{\prime}\left(x_{i}^{\prime}\right)^{\top}\right]^{1 / 2}(\hat{\Theta}-\Theta)\right\|_{2}^{k} \\
& +2^{3 k}(2 \epsilon)^{k-2} \lambda_{k}^{2 k}\left\|\hat{\Sigma}^{1 / 2}(\hat{\Theta}-\Theta)\right\|_{2}^{2 k} \\
& \left.+2^{3 k}(2 \epsilon)^{k-1} \lambda_{k}^{k} \mathbb{E}\left[\left(y_{i}^{*}-\left\langle x_{i}^{*}, \hat{\Theta}\right\rangle\right)^{2}\right]^{k / 2}\left\|\hat{\Sigma}^{1 / 2}(\hat{\Theta}-\Theta)\right\|_{2}^{k}\right\}
\end{aligned}
$$

Next, we relate the covariance of the samples indicated by $w$ to the covariance on the uncorrupted points. Observe, a real world proof of this follows simply from Fact 3.3.

Lemma 5.4 (Rounding). Consider the hypothesis of Theorem 5.1. Let $\hat{\Theta}=\arg \min _{\Theta} \frac{1}{n} \sum_{i \in[n]}\left(y_{i}^{*}-\left\langle x_{i}^{*}, \Theta\right\rangle\right)^{2}$ be the empirical loss minimizer on the uncorrupted samples. Then,

$$
\left\|\hat{\Sigma}^{1 / 2}(\hat{\Theta}-\tilde{\mathbb{E}}[\Theta])\right\|_{2} \leqslant O\left(\epsilon^{1-\frac{1}{k}} \lambda_{k}\right)\left(\mathbb{E}\left[\left(y_{i}^{*}-\left\langle x_{i}^{*}, \hat{\Theta}\right\rangle\right)^{2}\right]^{\frac{1}{2}}\right)
$$

Lemma 5.5 (Bounding Optimization ANd Generalization ERROR). Under the hypothesis of Theorem 5.1, for any $\zeta>0$, if $n \geqslant n_{0}$, such that $n_{0}=\Omega\left(\max \left\{c_{4} d / \zeta^{2}, d^{O(k)}\right\}\right)$, with probability at least $1-$ $1 / \operatorname{poly}(d), \mathbb{E}\left[y_{i}^{*}-\left\langle x_{i}^{*}, \hat{\Theta}\right\rangle^{2}\right]^{\frac{1}{2}} \leqslant(1+\zeta) \mathbb{E}_{x, y \sim \mathcal{D}}\left[y-\left\langle x, \Theta^{*}\right\rangle^{2}\right]^{\frac{1}{2}}$.
Algorithm 5.6 (Optimal Robust Regression in Polynomial Time).

Input: $n$ samples $\mathcal{X}_{\epsilon}$ from the robust regression model $\mathcal{R}_{\mathcal{D}}\left(\epsilon, \Theta^{*}, \Sigma^{*}\right)$.

\section{Operation:}

(1) Find a degree- $O(k)$ pseudo-distribution $\tilde{\zeta}$ satisfying $\mathcal{A}_{\epsilon, \lambda_{k}}$ and minimizing

$$
\min _{w, x^{\prime}, y^{\prime}, \Theta} \tilde{\mathbb{E}}_{\tilde{\zeta}}\left[\left(\frac{1}{n} \sum_{i \in[n]} w_{i}\left(y_{i}^{\prime}-\left\langle\Theta, x^{\prime}\right\rangle\right)^{2}\right)^{k}\right]
$$

(2) Round the pseudo-distribution to obtain an estima-

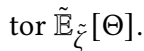

Output: A vector $\tilde{\mathbb{E}}_{\tilde{\zeta}}[\Theta]$ such that the recovery guarantee in Theorem 5.1 is satisfied.

Proof of Theorem 5.1. Given $n \geqslant n_{0}$ samples, it follows from Lemma 5.2 , that with probability $1-1 / \operatorname{poly}(d)$, the constraint system $\mathcal{A}_{\epsilon, \lambda_{k}}$ is feasible. Let $\xi_{1}$ be the event that the system is feasible and condition on it. Then, it follows from Lemma 5.4 and Lemma 5.5, with probability $1-1 / \operatorname{poly}(d)$,

$$
\left\|\hat{\Sigma}^{1 / 2}\left(\tilde{\mathbb{E}}_{\tilde{\zeta}}[\Theta]-\hat{\Theta}\right)\right\|_{2} \leqslant O\left(\lambda_{k} \epsilon^{1-1 / k}\right) \operatorname{err}_{\mathcal{D}}\left(\Theta^{*}\right)^{1 / 2}
$$

Let $\xi_{2}$ be the event that (25) holds and condition on it. It then follows from Fact 3.2, with probability $1-1 / \operatorname{poly}(d)$,

$$
\left\|\left(\Sigma^{*}\right)^{1 / 2}\left(\tilde{\mathbb{E}}_{\tilde{\zeta}}[\Theta]-\hat{\Theta}\right)\right\|_{2} \leqslant O\left(\lambda_{k} \epsilon^{1-1 / k}\right) \operatorname{err}_{\mathcal{D}}\left(\Theta^{*}\right)^{1 / 2}
$$

Let $\xi_{2}$ be the event that (26) holds and condition on it. It remains to relate the hyperplanes $\hat{\Theta}$ and $\Theta^{*}$. By reverse triangle inequality,

$$
\begin{aligned}
\left\|\left(\Sigma^{*}\right)^{1 / 2}\left(\tilde{\mathbb{E}}_{\tilde{\zeta}}[\Theta]-\Theta^{*}\right)\right\|_{2} & -\left\|\left(\Sigma^{*}\right)^{1 / 2}\left(\Theta^{*}-\hat{\Theta}\right)\right\|_{2} \\
& \leqslant\left\|\left(\Sigma^{*}\right)^{1 / 2}\left(\tilde{\mathbb{E}}_{\tilde{\zeta}}[\Theta]-\hat{\Theta}\right)\right\|_{2}
\end{aligned}
$$


Using normal equations, we have $\hat{\Theta}=\hat{\Sigma}^{-1} \mathbb{E}\left[x_{i} y_{i}\right]$ and $\Theta^{*}=$ $\left(\Sigma^{*}\right)^{-1} \mathbb{E}[x y]$. Since $\hat{\Sigma} \leq(1+0.01) \Sigma^{*}$,

$$
\begin{aligned}
\left\|\left(\Sigma^{*}\right)^{1 / 2}\left(\Theta^{*}-\hat{\Theta}\right)\right\|_{2} & =\left\|\left(\Sigma^{*}\right)^{1 / 2}\left(\hat{\Sigma}^{-1} \hat{\Sigma} \Theta^{*}-\hat{\Sigma}^{-1} \mathbb{E}\left[x_{i} y_{i}\right]\right)\right\|_{2} \\
& =\left\|\left(\Sigma^{*}\right)^{1 / 2} \hat{\Sigma}^{-1}\left(\mathbb{E}\left[x_{i}\left(y_{i}-x_{i}^{\top} \Theta^{*}\right)\right]\right)\right\|_{2} \\
& \leqslant 1.01\left\|\mathbb{E}\left[\left(\Sigma^{*}\right)^{-1 / 2} x_{i}\left(y_{i}-x_{i}^{\top} \Theta^{*}\right)\right]\right\|_{2}
\end{aligned}
$$

By Jensen's inequality $\mathbb{E}\left[\left\|\frac{1}{n} \sum_{i \in[n]}\left(\Sigma^{*}\right)^{-1 / 2} x_{i}\left(y_{i}-x_{i}^{\top} \Theta^{*}\right)\right\|_{2}\right] \leqslant$ $\sqrt{\mathbb{E}\left[\left\|\frac{1}{n} \sum_{i \in[n]}\left(\Sigma^{*}\right)^{-1 / 2} x_{i}\left(y_{i}-x_{i}^{\top} \Theta^{*}\right)\right\|_{2}^{2}\right]}$. Using negatively correlated moments, we have

$$
\mathbb{E}\left[\left(\Sigma^{*}\right)^{-1}(x)_{1}^{2}\left(y-x^{\top} \Theta^{*}\right)^{2}\right] \leqslant \mathbb{E}\left[\left(\Sigma^{*}\right)^{-1}(x)_{1}^{2}\right] \mathbb{E}\left[\left(y-x^{\top} \Theta^{*}\right)^{2}\right]
$$

Setting $v=\left(\Sigma^{*}\right)^{1 / 2} e_{1}$ and using Hypercontractivity of the covariates and the noise in the above equation,

$$
\mathbb{E}\left[\Sigma^{-1}(x)_{1}^{2}\right] \mathbb{E}\left[\left(y-x^{\top} \Theta^{*}\right)^{2}\right] \leqslant O\left(c_{2}^{2} \eta_{2}^{2}\right) \operatorname{err}_{\mathcal{D}}\left(\Theta^{*}\right)
$$

We can then conclude

$$
\mathbb{E}\left[\left\|\frac{1}{n} \sum_{i \in[n]}\left(\Sigma^{*}\right)^{-1 / 2} x_{i}\left(y_{i}-x_{i}^{\top} \Theta^{*}\right)\right\|_{2}\right] \leqslant O\left(c_{2} \eta_{2}\right) \sqrt{\frac{d \operatorname{err}_{\mathcal{D}}\left(\Theta^{*}\right)}{n}}
$$

Applying Chebyschev's Inequality yields the claim.

\section{LOWER BOUNDS}

In this section, we present information-theoretic lower bounds on the rate of convergence of parameter estimation and least-squares error for robust regression. Our constructions proceed by demonstrating two distributions over regression instances that are $\epsilon$-close in total variation distance and the marginal distribution over the covariates is hypercontractive, yet the true hyperplanes are $f(\epsilon)$-far in scaled $\ell_{2}$ distance. We defer the proofs to the full version.

True Linear Model. Consider the setting where there exists an optimal hyperplane $\Theta^{*}$ that is used to generate the data, with the addition of independent noise added to each sample, i.e. $y=\left\langle x, \Theta^{*}\right\rangle+\omega$, where $\omega$ is independent of $x$. Further, we assume that covariates and noise are hypercontractive. In this setting, Theorem 4.1 implies that we can recover a hyperplane close to $\Theta^{*}$ at a rate proportional to $\epsilon^{1-1 / k}$. We show that this dependence is tight for $k=4$. We note that independent noise is a special case of the distribution having negatively correlated moments.

Theorem 6.1 (True Linear Model Lower Bound, Theorem 1.9 RESTATED). For any $\epsilon>0$, there exist two distributions $\mathcal{D}_{1}, \mathcal{D}_{2}$ over $\mathbb{R}^{2} \times \mathbb{R}$ such that the marginal distribution over $\mathbb{R}^{2}$ has covariance $\Sigma$ and is $\left(c_{k}, k\right)$-hypercontractive yet $\left\|\Sigma^{1 / 2}\left(\Theta_{1}-\Theta_{2}\right)\right\|_{2}=$ $\Omega\left(\sqrt{c_{k}} \sigma \epsilon^{1-1 / k}\right)$, where $\Theta_{1}, \Theta_{2}$ be the optimal hyperplanes for $\mathcal{D}_{1}$ and $\mathcal{D}_{2}$ respectively, $\sigma=\max \left(\operatorname{err}_{\mathcal{D}_{1}}\left(\Theta_{1}\right), \operatorname{err}_{\mathcal{D}_{2}}\left(\Theta_{2}\right)\right)<1 / \epsilon^{1 / k}$ and the noise $\omega$ is uniform over $[-\sigma, \sigma]$.

Agnostic Model. Next, consider the setting where we simply observe samples from $(x, y) \sim \mathcal{D}$, and our goal is to return is to return the minimizer of the squared error, given by $\Theta^{*}=\mathbb{E}\left[x x^{\top}\right]^{-1} \mathbb{E}[x y]$. Here, the distribution of the noise is allowed to depend on the covariates arbitrarily. We further assume the noise is hypercontractive and obtain a lower bound proportional to $\epsilon^{1-2 / k}$ for recovering an estimator close to $\Theta^{*}$. This matches the upper boundd obtained in Corollary 4.2 .

Theorem 6.2 (Agnostic Model Lower Bound, Theorem 1.11 RESTATED). For any $\epsilon>0$, there exist two distributions $\mathcal{D}_{1}, \mathcal{D}_{2}$ over $\mathbb{R}^{2} \times \mathbb{R}$ such that the marginal distribution over $\mathbb{R}^{2}$ has covariance $\Sigma$ and is $\left(c_{k}, k\right)$-hypercontractive yet $\left\|\Sigma^{1 / 2}\left(\Theta_{1}-\Theta_{2}\right)\right\|_{2}=$ $\Omega\left(\sqrt{c_{k}} \sigma \epsilon^{1-2 / k}\right)$, where $\Theta_{1}, \Theta_{2}$ be the optimal hyperplanes for $\mathcal{D}_{1}$ and $\mathcal{D}_{2}$ respectively, $\sigma=\max \left(\operatorname{err}_{\mathcal{D}_{1}}\left(\Theta_{1}\right), \operatorname{err}_{\mathcal{D}_{2}}\left(\Theta_{2}\right)\right)<1 / \epsilon^{1 / k}$ and the noise is a function of the marginal distribution of $\mathbb{R}^{2}$.

Bounded Covariance Distributions. In the heavy-tailed setting, the minimal assumption is to consider a distribution over the covariates with bounded covariance. In this setting, we show that robust estimators for linear regression do not exist, even when the underlying linear model has no noise, i.e. the uncorrupted samples are drawn as follows: $y_{i}=\left\langle\Theta^{*}, x_{i}\right\rangle$.

Theorem 6.3 (Lower Bound for Bounded Covariance DisTRIBUTIONS). For all $\epsilon>0$, there exist two distributions $\mathcal{D}_{1}, \mathcal{D}_{2}$ over $\mathbb{R} \times \mathbb{R}$ corresponding to the linear model $y=\left\langle\Theta_{1}, x\right\rangle$ and $y=\left\langle\Theta_{2}, x\right\rangle$ respectively, such that the marginal distribution over $\mathbb{R}$ has variance $\sigma$ and $d_{T V}\left(\mathcal{D}_{1}, \mathcal{D}_{2}\right) \leqslant \epsilon$, yet $\left|\sigma\left(\Theta_{1}-\Theta_{2}\right)\right|=\Omega(\sigma)$.

\section{ACKNOWLEDGMENTS}

We thank Sivaraman Balakrishnan, Sam Hopkins, Pravesh Kothari, Jerry Li, Pradeep Ravikumar and David Wajc for illuminating discussions related to this project. In particular, we thank Pravesh Kothari for answering several technical questions regarding lower bounds appearing in a previous version of [33]. A.B. would like to thank the partial support from the Office of Naval Research (ONR) grant N00014-18-1-2562, and the National Science Foundation (NSF) under Grant No. CCF-1815840.

\section{REFERENCES}

[1] Ainesh Bakshi and Pravesh Kothari. 2020. List-Decodable Subspace Recovery via Sum-of-Squares. arXiv preprint arXiv:2002.05139 (2020).

[2] Ainesh Bakshi and Pravesh Kothari. 2020. Outlier-Robust Clustering of NonSpherical Mixtures. arXiv preprint arXiv:2005.02970 (2020).

[3] Boaz Barak. [n.d.]. Proofs, beliefs, and algorithms through the lens of sum-ofsquares. ([n.d.]).

[4] Boaz Barak, Jonathan A. Kelner, and David Steurer. 2015. Dictionary Learning and Tensor Decomposition via the Sum-of-Squares Method. In STOC. ACM, 143-151.

[5] Kush Bhatia, Prateek Jain, Parameswaran Kamalaruban, and Purushottam Kar. 2017. Consistent robust regression. In Advances in Neural Information Processing Systems. 2110-2119.

[6] Kush Bhatia, Prateek Jain, and Purushottam Kar. 2015. Robust regression via hard thresholding. In Advances in Neural Information Processing Systems. 721-729.

[7] Vijay V. S. P. Bhattiprolu, Venkatesan Guruswami, and Euiwoong Lee. 2017. Sum-of-Squares Certificates for Maxima of Random Tensors on the Sphere. In APPROX-RANDOM (LIPIcs, Vol. 81). Schloss Dagstuhl - Leibniz-Zentrum fuer Informatik, 31:1-31:20. 
[8] Moses Charikar, Jacob Steinhardt, and Gregory Valiant. 2017. Learning from untrusted data. In STOC. ACM, 47-60.

[9] Yu Cheng, Ilias Diakonikolas, and Rong Ge. 2019. High-Dimensional Robust Mean Estimation in Nearly-Linear Time. In Proceedings of the Thirtieth Annual ACM-SIAM Symposium on Discrete Algorithms, SODA 2019, San Diego, California, USA, fanuary 6-9, 2019, Timothy M. Chan (Ed.). SIAM, 2755-2771. https://doi org/10.1137/1.9781611975482.171

[10] Yu Cheng, Ilias Diakonikolas, Rong Ge, and David P. Woodruff. 2019. Faster Algorithms for High-Dimensional Robust Covariance Estimation. In Conference on Learning Theory, COLT 2019, 25-28 fune 2019, Phoenix, AZ, USA (Proceedings of Machine Learning Research, Vol. 99), Alina Beygelzimer and Daniel Hsu (Eds.) PMLR, 727-757. http://proceedings.mlr.press/v99/cheng19a.html

[11] Yeshwanth Cherapanamjeri, Samuel B. Hopkins, Tarun Kathuria, Prasad Raghavendra, and Nilesh Tripuraneni. 2020. Algorithms for Heavy-Tailed Statistics: Regression, Covariance Estimation, and Beyond. In Proceedings of the 52nd Annual ACM SIGACT Symposium on Theory of Computing (Chicago, IL, USA) (STOC 2020). Association for Computing Machinery, New York, NY, USA 601-609. https://doi.org/10.1145/3357713.3384329

[12] Yeshwanth Cherapanamjeri, Sidhanth Mohanty, and Morris Yau. 2020. List Decodable Mean Estimation in Nearly Linear Time. arXiv preprint arXiv:2005.09796 (2020).

[13] Ilias Diakonikolas, Samuel B Hopkins, Daniel Kane, and Sushrut Karmalkar 2020. Robustly Learning any Clusterable Mixture of Gaussians. arXiv preprint arXiv:2005.06417 (2020)

[14] Ilias Diakonikolas, Gautam Kamath, Daniel Kane, Jerry Li, Jacob Steinhardt, and Alistair Stewart. 2019. Sever: A Robust Meta-Algorithm for Stochastic Optimization. In Proceedings of the 36th International Conference on Machine Learning, ICML 2019, 9-15 June 2019, Long Beach, California, USA (Proceedings of Machine Learning Research, Vol. 97), Kamalika Chaudhuri and Ruslan Salakhutdinov (Eds.) PMLR, 1596-1606. http://proceedings.mlr.press/v97/diakonikolas19a.html

[15] Ilias Diakonikolas, Gautam Kamath, Daniel M Kane, Jerry Li, Ankur Moitra and Alistair Stewart. 2016. Robust estimators in high dimensions without the computational intractability. In Foundations of Computer Science (FOCS), 2016 IEEE 57th Annual Symposium on. IEEE, 655-664.

[16] Ilias Diakonikolas, Gautam Kamath, Daniel M. Kane, Jerry Li, Ankur Moitra, and Alistair Stewart. 2017. Being Robust (in High Dimensions) Can Be Practical. In ICML (Proceedings of Machine Learning Research, Vol. 70). PMLR, 999-1008.

[17] Ilias Diakonikolas, Gautam Kamath, Daniel M. Kane, Jerry Li, Ankur Moitra, and Alistair Stewart. 2018. Robustly Learning a Gaussian: Getting Optimal Error Efficiently. In Proceedings of the Twenty-Ninth Annual ACM-SIAM Symposium on Discrete Algorithms, SODA 2018, New Orleans, LA, USA, January 7-10, 2018, Artur Czumaj (Ed.). SIAM, 2683-2702. https://doi.org/10.1137/1.9781611975031.171

[18] Ilias Diakonikolas, Gautam Kamath, Daniel M Kane, Jerry Li, Jacob Steinhardt, and Alistair Stewart. 2018. Sever: A Robust Meta-Algorithm for Stochastic Optimization. arXiv preprint arXiv:1803.02815 (2018).

[19] Ilias Diakonikolas and Daniel M Kane. 2019. Recent Advances in Algorithmic High-Dimensional Robust Statistics. arXiv preprint arXiv:1911.05911 (2019).

[20] Ilias Diakonikolas, Daniel M. Kane, and Alistair Stewart. 2017. Statistical Query Lower Bounds for Robust Estimation of High-Dimensional Gaussians and Gaussian Mixtures. In FOCS. IEEE Computer Society, 73-84.

[21] Ilias Diakonikolas, Weihao Kong, and Alistair Stewart. 2018. Efficient algorithms and lower bounds for robust linear regression. arXiv preprint arXiv:1806.00040 (2018).

[22] Ilias Diakonikolas, Weihao Kong, and Alistair Stewart. 2019. Efficient Algorithms and Lower Bounds for Robust Linear Regression. In Proceedings of the Thirtieth Annual ACM-SIAM Symposium on Discrete Algorithms, SODA 2019, San Diego, California, USA, January 6-9, 2019, Timothy M. Chan (Ed.). SIAM, 2745-2754.

[23] Noah Fleming, Pravesh Kothari, Toniann Pitassi, et al. 2019. Semialgebraic Proofs and Efficient Algorithm Design. now the essence of knowledge.

[24] M. Grötschel, L. Lovász, and A. Schrijver. 1981. The ellipsoid method and its consequences in combinatorial optimization. Combinatorica 1, 2 (1981), 169-197. https://doi.org/10.1007/BF02579273

[25] Samuel B Hopkins. 2018. Sub-Gaussian Mean Estimation in Polynomial Time. arXiv preprint arXiv:1809.07425 (2018)

[26] Samuel B Hopkins and Jerry Li. 2018. Mixture models, robustness, and sum of squares proofs. In Proceedings of the 50th Annual ACM SIGACT Symposium on Theory of Computing. 1021-1034.

[27] Samuel B Hopkins, Tselil Schramm, and Jonathan Shi. 2019. A Robust Spectral Algorithm for Overcomplete Tensor Decomposition. In Conference on Learning Theory. 1683-1722.

[28] Peter J Huber. 1964. Robust estimation of a location parameter. The Annals of Mathematical Statistics 35, 1 (1964), 73-101.

[29] Peter J Huber. 2011. Robust statistics. In International Encyclopedia of Statistical Science. Springer, 1248-1251.

[30] Louis A Jaeckel. 1972. Estimating regression coefficients by minimizing the dispersion of the residuals. The Annals of Mathematical Statistics (1972), 14491458.
[31] Sushrut Karmalkar, Adam Klivans, and Pravesh Kothari. 2019. List-decodable linear regression. In Advances in Neural Information Processing Systems. 74237432.

[32] Adam Klivans, Pravesh K Kothari, and Raghu Meka. 2018. Efficient Algorithms for Outlier-Robust Regression. arXiv preprint arXiv:1803.03241 (2018).

[33] Adam R. Klivans, Pravesh K. Kothari, and Raghu Meka. 2018. Efficient Algorithms for Outlier-Robust Regression. In Conference On Learning Theory, COLT 2018, Stockholm, Sweden, 6-9 fuly 2018. 1420-1430. http://proceedings.mlr.press/v75/ klivans18a.html

[34] Pravesh K. Kothari and Jacob Steinhardt. 2017. Better Agnostic Clustering via Relaxed Tensor Norms. (2017)

[35] Pravesh K. Kothari and David Steurer. 2017. Outlier-robust moment-estimation via sum-of-squares. CoRR abs/1711.11581 (2017). arXiv:1711.11581 http://arxiv. org/abs/1711.11581

[36] Pravesh K Kothari and David Steurer. 2017. Outlier-robust moment-estimation via sum-of-squares. arXiv preprint arXiv:1711.11581 (2017).

[37] Kevin A Lai, Anup B Rao, and Santosh Vempala. 2016. Agnostic estimation of mean and covariance. In Foundations of Computer Science (FOCS), 2016 IEEE 57th Annual Symposium on. IEEE, 665-674.

[38] Jean B. Lasserre. 2001. New positive semidefinite relaxations for nonconvex quadratic programs. In Advances in convex analysis and global optimization (Pythagorion, 2000). Nonconvex Optim. Appl., Vol. 54. Kluwer Acad. Publ., Dordrecht, 319-331. https://doi.org/10.1007/978-1-4613-0279-7_18

[39] Jerry Zheng Li. 2018. Principled approaches to robust machine learning and beyond. Ph.D. Dissertation. Massachusetts Institute of Technology.

[40] Tengyu Ma, Jonathan Shi, and David Steurer. 2016. Polynomial-Time Tensor Decompositions with Sum-of-Squares. In FOCS. IEEE Computer Society, 438-446.

[41] Yurii Nesterov. 2000. Squared functional systems and optimization problems. In High performance optimization. Appl. Optim., Vol. 33. Kluwer Acad. Publ., Dordrecht, 405-440. https://doi.org/10.1007/978-1-4757-3216-0_17

[42] Pablo A Parrilo. 2000. Structured semidefinite programs and semialgebraic geometry methods in robustness and optimization. Ph.D. Dissertation. California Institute of Technology.

[43] Adarsh Prasad, Arun Sai Suggala, Sivaraman Balakrishnan, and Pradeep Ravikumar. 2020. Robust estimation via robust gradient estimation. Fournal of the Royal Statistical Society: Series B (Statistical Methodology) 82, 3 (2020), 601-627. https://doi.org/10.1111/rssb.12364 arXiv:https://rss.onlinelibrary.wiley.com/doi/pdf/10.1111/rssb.12364

[44] Prasad Raghavendra, Satish Rao, and Tselil Schramm. 2017. Strongly refuting random CSPs below the spectral threshold. In STOC. ACM, 121-131.

[45] Prasad Raghavendra, Tselil Schramm, and David Steurer. 2018. High-dimensional estimation via sum-of-squares proofs. arXiv preprint arXiv:1807.11419 6 (2018).

[46] Prasad Raghavendra and Morris Yau. 2020. List decodable learning via sum of squares. In Proceedings of the Fourteenth Annual ACM-SIAM Symposium on Discrete Algorithms. SIAM, 161-180.

[47] Prasad Raghavendra and Morris Yau. 2020. List Decodable Subspace Recovery. arXiv:2002.03004 [cs.DS]

[48] Peter Rousseeuw and Victor Yohai. 1984. Robust regression by means of Sestimators. In Robust and nonlinear time series analysis. Springer, 256-272.

[49] Peter J Rousseeuw. 1984. Least median of squares regression. Fournal of the American statistical association 79, 388 (1984), 871-880.

[50] Tselil Schramm and David Steurer. 2017. Fast and robust tensor decomposition with applications to dictionary learning. In COLT (Proceedings of Machine Learning Research, Vol. 65). PMLR, 1760-1793.

[51] Pranab Kumar Sen. 1968. Estimates of the regression coefficient based on Kendall's tau. Fournal of the American statistical association 63, 324 (1968), 13791389.

[52] N. Z. Shor. 1987. Quadratic optimization problems. Izv. Akad. Nauk SSSR Tekhn. Kibernet. 1 (1987), 128-139, 222.

[53] Jacob Steinhardt. 2018. ROBUST LEARNING: INFORMATION THEORY AND ALGORITHMS. Ph.D. Dissertation. STANFORD UNIVERSITY.

[54] Jacob Steinhardt, Moses Charikar, and Gregory Valiant. 2017. Resilience: A Criterion for Learning in the Presence of Arbitrary Outliers. CoRR abs/1703.04940 (2017)

[55] Arun Sai Suggala, Kush Bhatia, Pradeep Ravikumar, and Prateek Jain. 2019. Adaptive hard thresholding for near-optimal consistent robust regression. arXiv preprint arXiv:1903.08192 (2019).

[56] Henri Theil. 1992. A rank-invariant method of linear and polynomial regression analysis. In Henri Theil's contributions to economics and econometrics. Springer, 345-381.

[57] Sanford Weisberg. 2005. Applied linear regression. Vol. 528. John Wiley \& Sons.

[58] Banghua Zhu, Jiantao Jiao, and Jacob Steinhardt. 2019. Generalized resilience and robust statistics. arXiv preprint arXiv:1909.08755 (2019).

[59] Banghua Zhu, Jiantao Jiao, and Jacob Steinhardt. 2020. Robust estimation via generalized quasi-gradients. arXiv preprint arXiv:2005.14073 (2020) 